\title{
Coherent optical OFDM: has its time come? [Invited]
}

\author{
William Shieh,* Xingwen Yi, Yiran Ma, and Qi Yang \\ Australian Research Council (ARC) Special Research Centre for Ultra-Broadband \\ Information Networks and National ICT Australia, Department of Electrical and \\ Electronic Engineering, The University of Melbourne, Melbourne, \\ Victoria 3010, Australia \\ *Corresponding author: w.shieh@ee.unimelb.edu.au
}

\begin{abstract}
Received November 30, 2007; revised January 12, 2008; accepted January 15, 2008; published February 29, 2008 (Doc. ID 90380)

There has been growing interest in coherent optical orthogonal frequencydivision multiplexing $(\mathrm{CO}-\mathrm{OFDM})$. We aim to present the case that the time for CO-OFDM has come, in terms of the demand from today's ever-advancing optical networks and the availability of its underlying technologies. We first lay out the signal processing aspect for CO-OFDM and then show a $2 \times 2$ multiple-input-multiple-output (MIMO) OFDM representation for the singlemode fiber optical channel. Through numerical simulation and experimental demonstration, we further present and discuss various MIMO-OFDM systems focusing on their polarization-mode dispersion resilience. Among those systems, the $2 \times 1$ MIMO-OFDM configuration incorporating polarization-time coding shows promise for optical access networks and broadcast networks. Finally, another class of the frequency-domain equalization techniques, namely, incoherent or coherent optical single-carrier frequency-domain equalization (CO-SCFDE) is discussed and experimentally demonstrated. (C) 2008 Optical Society of America

OCIS codes: $060.0060,060.1660,060.5060$.
\end{abstract}

\section{Introduction}

Coherent optical orthogonal frequency-division multiplexing (CO-OFDM) has recently emerged as an attractive modulation format for optical communications [1], and significant progress has been made through theoretical analysis and experimental demonstration [2-4]. CO-OFDM brings to optical communications the combination of two powerful techniques, coherent optical detection and orthogonal frequency-division multiplexing (OFDM). From the perspective of coherent optical detection in optical communications, the first wave of the intense interest appeared in the 1980s and early $1990 \mathrm{~s}$, when the coherent detection was viewed as a promising technique to improve the receiver sensitivity [5-7]. However, the ensuing invention of the erbiumdoped fiber amplifier (EDFA) reduced research on coherent communication to peripheral interest. In the early 21 st century, the impressive record-performance experimental demonstration using a differential-phase-shift-keying (DPSK) system [8], in spite of an incoherent form of modulation by itself, reignited the interest in coherent communications. The second wave of research on coherent communications is highlighted by the remarkable theoretical and experimental demonstrations from various groups around the world [9-14]. It is rather instructive to point out that the circumstances and the underlying technologies for the current drive for coherent communications are entirely different from those of a decade ago, thanks to the rapid technological advancement within the past decade in various fields. First, current coherent detection systems are heavily entrenched in silicon-based digital signal processing for highspeed signal phase estimation and channel equalization. Second, multicarrier technology, which has emerged and thrived in the RF domain during the past decade, has gradually encroached into the optical domain [1-4,15-20]. Third, in contrast to the optical system that was dominated by a low-speed, point-to-point, and single-channel system a decade ago, modern optical communication systems have advanced to massive wave-division-multiplexed (WDM) and reconfigurable optical networks with a transmission speed approaching $100 \mathrm{Gbits} / \mathrm{s}$. In a nutshell, the primary aim of coherent communications has shifted toward supporting these high-speed dynamic networks by simplifying the network installation, monitoring, and maintenance. 
OFDM has emerged as the leading physical-layer interface in wireless communications in the past decade. It is a special form of a broader class of multicarrier modulation where a data stream is carried with many lower-rate subcarrier tones [21]. OFDM has been widely studied in mobile communications to combat hostile frequency-selective fading and has been incorporated into wireless network standards (802.11a/g WiFi, HiperLAN2, 802.16 WiMAX) and digital audio and video broadcasting (DAB and DVB-T) in Europe, Asia, Australia, and other parts of the world.

The synergies between coherent optical communications and OFDM are twofold. The coherent system brings OFDM a much needed linearity in RF-to-optical (RTO) upconversion and optical-to-RF (OTR) downconversion. OFDM brings coherent system computation efficiency and ease of channel and phase estimation. In spite of the fact that all the current CO-OFDM experimental demonstrations use off-line signal processing $[3,4,13,14]$, the complementary metal-oxide semiconductor (CMOS) application-specific integrated circuit (ASIC) chips recently demonstrated for singlecarrier coherent systems [22,23] signify that the current silicon speed can support $40 \mathrm{Gbit} / \mathrm{s}$ OFDM transmission systems. Because of its superior scalability with the bit rate of the transmission systems, CO-OFDM is well-positioned to be an attractive choice of modulation format for the next generation of $100 \mathrm{Gbit} / \mathrm{s}$ transmission.

In this paper, we review the progress on CO-OFDM systems as well as present the latest research results. We first lay out the signal processing aspect of the CO-OFDM and then show a $2 \times 2$ multiple-input-multiple-output (MIMO) OFDM representation for the single-mode fiber optical channel. We further show and discuss the experimental demonstrations for various MIMO-OFDM configurations. In particular, we carry out long-haul transmission for the $2 \times 2$ and $1 \times 2$ MIMO-OFDM systems and shortreach transmission for the $2 \times 1$ MIMO-OFDM systems that may have promise for optical broadcasting networks. Finally, another class of the frequency-domain equalization (FDE) techniques, namely, incoherent or coherent optical single-carrier frequency-domain equalization (IO-SCFDE or CO-SCFDE) is discussed and demonstrated. As such, this paper focuses on signal processing, experimentation, and the underlying technologies of CO-OFDM, serving as a complement to our recent invited paper [24], which concentrates on theory and design.

\section{Signal Processing for CO-OFDM}

Figure 1 shows the conceptual diagram of a generic CO-OFDM system including five
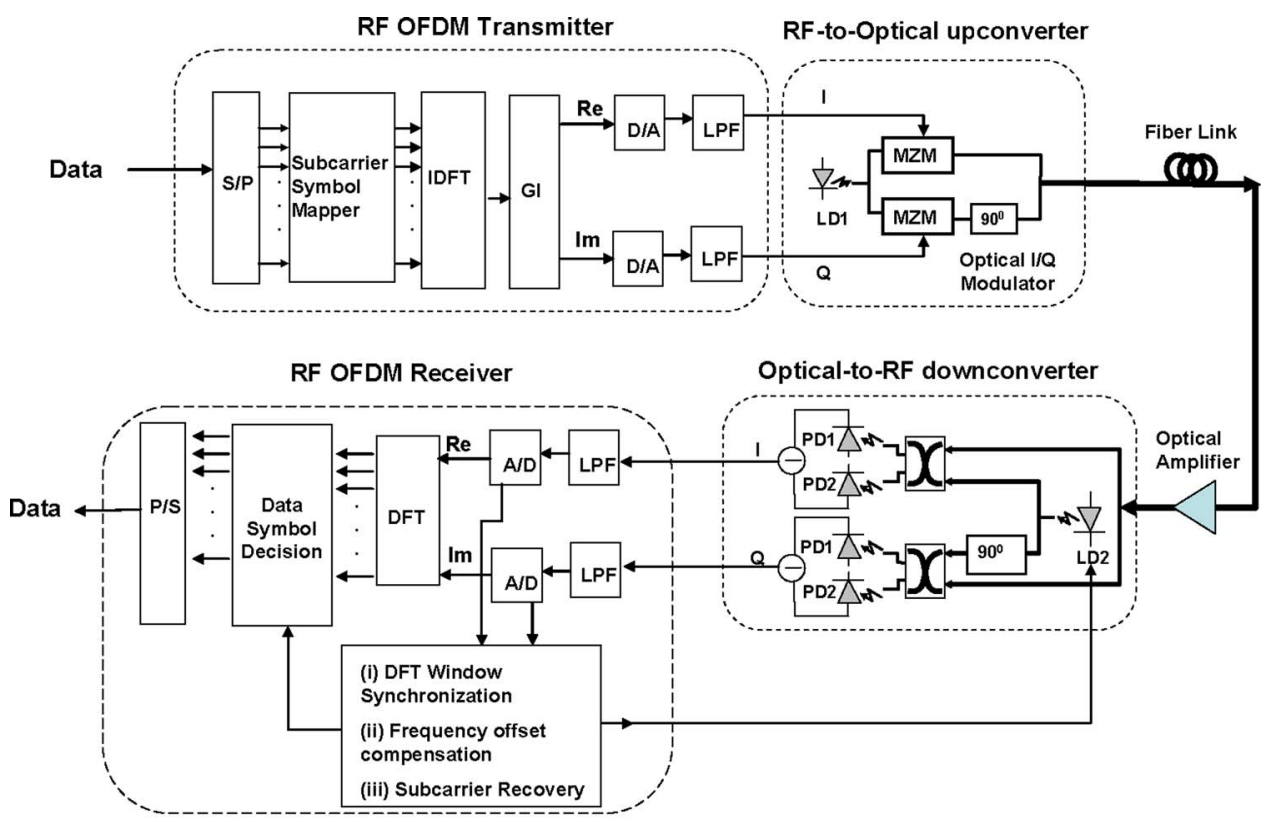

S/P: Serial-to-parallel GI: Guard Time Insertion D/A: Digital-to-Analog (I)DFT: (Inverse) Discrete Fourier Transform LPF: Low-Pass Filter BPF: Bandpass Filter MZM: Mach-Zenhder Modulator OBPF: Optical Bandpass Filter PD: Photodiode LD: Laser Diode

Fig. 1. Conceptual diagram for a generic CO-OFDM system with direct up-down conversion architecture. 
basic functional blocks [1,25]: RF OFDM transmitter, RTO upconverter, optical link, OTR downconverter, and RF OFDM receiver. In this paper, the term RF is used interchangeably with electrical to signify the physical interface in contrast with that in the optical domain. The transmission channel linearity is the fundamental assumption for the OFDM technique. Therefore, the study of the nonlinearity for each functional block is of critical importance. The RF OFDM transmitter and receiver have been studied in RF systems [21] and thus will hold the same importance to CO-OFDM systems. One unique aspect of the CO-OFDM investigation is that it brings new sources of nonlinearity, including RTO upconversion [25], OTR downconversion, and fiber link transmission [26]. In this section, we will concentrate on the signal processing aspect of CO-OFDM and therefore assume perfect linearity in each functional block.

We will trace the signal flow end-to-end and illustrate the signal processing that arises in each functional block. In the RF OFDM transmitter, the input digital data is first converted from serial to parallel into a block of bits consisting of $N_{s c}$ information symbols; each may comprise multiple bits for $m$-ary coding. This information symbol will be mapped onto the two-dimensional complex signal $c_{k i}$, for instance, using Gray coding, where $c_{k i}$ stands for the mapped complex information symbol. The subscripts of $c_{k i}$ correspond to the sequence of the subcarriers and OFDM blocks, which will be further defined below. The time-domain OFDM signal is obtained through inverse discrete Fourier transform (IDFT) of $c_{k i}$, and a guard interval is inserted to avoid the channel dispersion [21]. The resultant baseband time-domain signal can be described as

$$
\begin{gathered}
s_{B}(t)=\sum_{i=-\infty}^{+\infty} \sum_{k=-N_{s c} / 2+1}^{k=N_{s c} / 2} c_{k i} \Pi\left(t-i T_{s}\right) \exp \left(j 2 \pi f_{k}\left(t-i T_{s}\right),\right. \\
f_{k}=\frac{k-1}{t_{s}}, \\
\Pi(t)= \begin{cases}1, & \left(-\Delta_{G}<t \leq t_{s}\right) \\
0, & \left(t \leq-\Delta_{G}, t>t_{s}\right)\end{cases}
\end{gathered}
$$

where $c_{k i}$ is the $i$ th information symbol at the $k$ th subcarrier; $f_{k}$ is the frequency of the subcarrier; $N_{s c}$ is the number of OFDM subcarriers; $T_{s}, \Delta_{G}$, and $t_{s}$ are the OFDM symbol period, guard interval length, and observation period, respectively; and $\Pi(t)$ is the rectangular pulse waveform of the OFDM symbol. The digital signal is then converted to analog form through a digital-to-analog converter (DAC) and filtered with a lowpass filter (LPF) to remove the alias sideband signal. The baseband OFDM signal can be further converted to the RF band through a RF in-phase/quadrature (I/Q) mixer (not shown). Figure 1 shows the direct upconversion architecture where the RF OFDM transmitter outputs a baseband OFDM signal. The subsequent RTO upconverter transforms the baseband signal to the optical domain using an optical I/Q modulator comprising a pair of Mach-Zehnder modulators (MZMs) with a $90^{\circ}$ phase offset. The baseband OFDM signal is directly upconverted to the optical domain given by

$$
E(t)=\exp \left(j \omega_{L D 1} t+\phi_{L D 1}\right) s_{B}(t)
$$

where $\omega_{L D 1}$ and $\phi_{L D 1}$, respectively, are the angular frequency and phase of the transmitter laser. The upconverted signal $E(t)$ traverses the optical medium with impulse response $h(t)$, and the received optical signal becomes

$$
E(t)=\exp \left(j \omega_{L D 1} t+\phi_{L D 1}\right) s_{B}(t) \otimes h(t)
$$

where $\otimes$ stands for convolution. The optical OFDM signal is then fed into the OTR downconverter where the optical OFDM signal is converted to a RF OFDM signal. Figure 1 shows the direct downconversion architecture where the intermediate frequency (IF) is near DC. The directly downconverted near-DC signal can be expressed as

$$
r(t)=\exp \left(j \omega_{\mathrm{off}} t+\Delta \phi\right) r_{0}(t), \quad r_{0}(t)=s_{B}(t) \otimes h(t)
$$




$$
\omega_{\mathrm{off}}=\omega_{L D 1}-\omega_{L D 2}, \quad \Delta \phi=\phi_{L D 1}-\phi_{L D 2},
$$

where $\Delta \omega_{\text {off }}$ and $\Delta \phi$ are, respectively, the angular frequency offset and phase offset between the transmit and receive lasers.

In the RF OFDM receiver, the downconverted near-DC OFDM signal is first sampled with an analog-to-digital converter (ADC). Then the signal needs to go through sophisticated three-level synchronization before the symbol decision can be made. The three levels of synchronization are as follows:

(i) Discrete Fourier transform (DFT) window synchronization where the OFDM symbol is properly delineated to avoid intersymbol interference.

(ii) Frequency synchronization, namely, frequency offset $\omega_{\text {off }}$ needs to be estimated, compensated, and, preferably, adjusted to a small value at the start.

(iii) The subcarrier recovery, where each subcarrier channel is estimated and compensated.

The detailed discussion of these three synchronizations will be presented in Subsections 2.B and 2.C. Assuming successful completion of DFT window synchronization and frequency synchronization, the RF OFDM signal through the DFT of the sampled value of Eq. (6) becomes

$$
r_{k i}=e^{\phi_{i}} h_{k i} c_{k i}+n_{k i}
$$

where $r_{k i}$ is the received information symbol, $\phi_{i}$ is the OFDM symbol phase (OSP) or common phase error (CPE), $h_{k i}$ is the frequency domain channel transfer function, and $n_{k i}$ is the noise. The third synchronization of the subcarrier recovery involves estimation of the OSP, $\phi_{i}$, and the channel transfer function, $h_{k i}$. Once they are known, an estimated value of $c_{k i}, \hat{c}_{k i}$ is given by the zero-forcing method as

$$
\hat{c}_{k i}=\frac{h_{k i}^{*}}{\left|h_{k i}\right|^{2}} e^{-i \phi_{i}} r_{k i} \text {. }
$$

$\hat{c}_{k i}$ is used for symbol decision or to recover the transmitted value $c_{k i}$, which is subsequently mapped back to the original transmitted digital bits.

The above terse description of CO-OFDM signal processing has so far left out the pilot-subcarrier or pilot-symbol insertion where a proportion of the subcarriers or all the subcarriers in one OFDM symbol are known values to the receiver. The purpose of these pilot subcarriers or symbols is to assist the above-mentioned three-level synchronization. Another important aspect of the CO-OFDM signal processing that has not been discussed is the error-correction coding involving the error-correction encoder-decoder and the interleaver-deinterleaver [21,27].

\section{A. DFT Window Synchronization}

Synchronization is one of the most critical functionalities for a CO-OFDM receiver. As discussed above, it can be divided into three levels of synchronization: DFT window timing synchronization, carrier-frequency offset synchronization, and subcarrier recovery. Figure 2 shows the time-domain structure of an OFDM signal consisting of many OFDM symbols. Each OFDM symbol is comprised of a guard interval and an observation period. It is imperative that the start of the DFT window, i.e., the observation period, is determined properly, as an improper DFT window will result in intersymbol interference (ISI) and intercarrier interference (ICI) [28].

A popular method of window synchronization was proposed by Schmidl and Cox [29]. In this method, a pilot symbol or preamble is transmitted consisting of two identical segments, as shown in Fig. 3, that can be expressed as

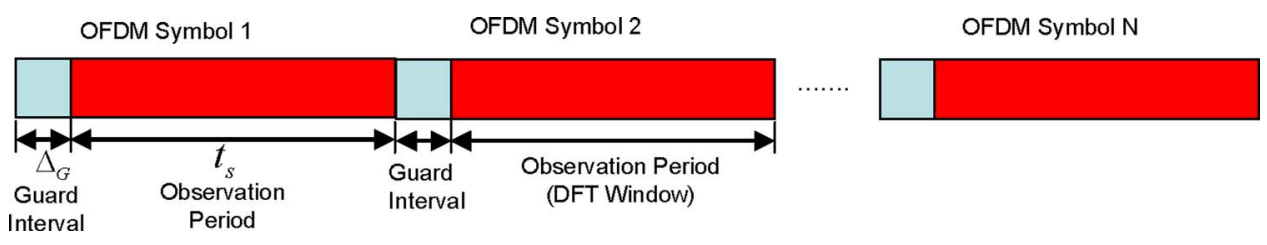

Fig. 2. Time-domain structure of an OFDM signal. 


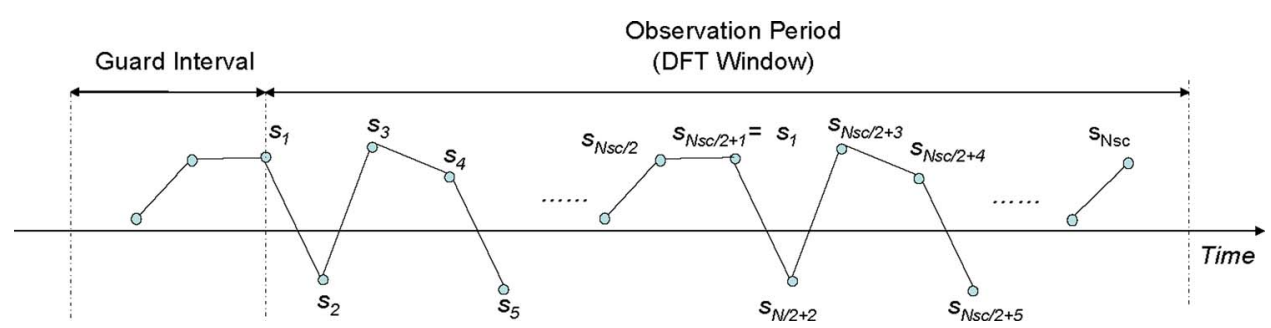

Fig. 3. Schmidl synchronization format.

$$
s_{m}=s_{m-N_{s c} / 2}, \quad m \in\left[N_{s c} / 2+1, N_{s c}\right],
$$

where $s_{m}$ is the $m$ th digital signal with a random value, when $m \in\left[1, N_{s c} / 2\right]$. Assuming a time-invariant channel transfer function $h(t)$, from Eq. (6) the sampled received signal will have the form

$$
r_{m}=r\left(m t_{s} / N_{s c}\right)=r_{m}^{0} e^{j \omega_{\text {off }} m t_{s} / N_{s c}}+n_{m} .
$$

We have assumed that the constant phase crosses the entire OFDM symbol or $\Delta \phi$ equals 0 in Eq. (6). The delineation can be identified by studying the following correlation function [27]:

$$
R_{d}=\sum_{m=1}^{N_{s c} / 2} r_{m+d}^{*} r_{m+d+N_{s c} / 2}
$$

The principle is that because the second half of $r_{m}$ is identical to the first half except for a phase shift. Assuming the frequency offset $\omega_{\text {off }}$ is small to start with, we anticipate that when $d=0$, the correlation function $R_{d}$ reaches its maximum value. The correlation function can be normalized to its maximum value given by

$$
M(d)=\left|R_{d} / S_{d}\right|^{2}, \quad S_{d}=\sqrt{\left(\sum_{m=1}^{N_{s c} / 2}\left|r_{m+d}^{2}\right|\right)\left(\sum_{m=1}^{N_{s c} / 2}\left|r_{m+d+N_{s c} / 2}^{2}\right|\right)} .
$$

$M(d)$ is defined as the DFT window synchronization timing metric. The timing metric takes the maximum value at $d=0$, namely, at the correct timing.

We have conducted a Monte Carlo simulation to confirm the DFT window synchronization using the format of Schmidl and Cox [29] for a CO-OFDM system at $10 \mathrm{Gbits} / \mathrm{s}$ under the influence of chromatic dispersion. The OFDM system parameters used for the simulation are a symbol period of $25.6 \mathrm{~ns}$, a guard time of $3.2 \mathrm{~ns}$, and a number of subcarriers of 256. Binary-phase-shift-keying (BPSK) encoding is used for each subcarrier resulting in a total bit rate of $10 \mathrm{Gbits} / \mathrm{s}$. The linewidths of the transmitter and receiver lasers are assumed to be $100 \mathrm{KHz}$ each, which is close to the value achieved with commercially available external-cavity semiconductor lasers [30]. The optical link noise from the optical amplifiers is assumed to be white Gaussian noise and the phase noise of the laser is modeled as white frequency noise characterized by its linewidth.

Figure 4(a) shows that the timing metric decreases from an ideal value of 1 to $\sim 0.7$ when the optical-to-signal-noise ratio (OSNR) is $5 \mathrm{~dB}$. For reference, to achieve a bit error rate (BER) of $10^{-3}$, an OSNR of $3.5 \mathrm{~dB}$ is needed. Both curves at an OSNR of

(a)

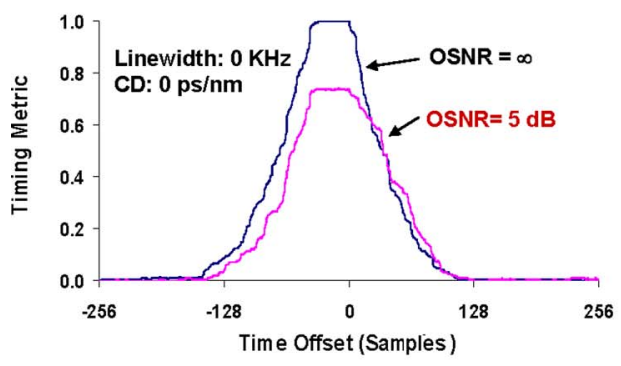

(b)

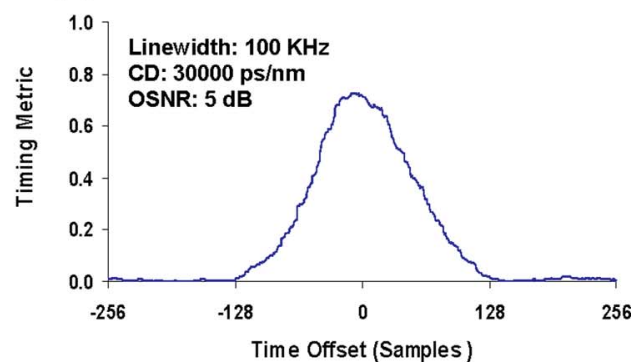

Fig. 4. Timing metric for a CO-OFDM system at the chromatic dispersion of (a) 0 and (b) $30,000 \mathrm{ps} / \mathrm{nm}$. 
infinity and $5 \mathrm{~dB}$ show the flat platform of 32 samples corresponding to a $3.2 \mathrm{~ns}$ guard time under no chromatic dispersion. However, at the chromatic dispersion of $30,000 \mathrm{ps} / \mathrm{nm}$ [Fig. 4(b)], the flat platform has almost disappeared, indicating that most of the guard interval has been affected by the cross talk from the neighboring OFDM symbols. Another consequence is that the timing metric peak is not obvious any more and multiple peaks may coexist in the timing metric diagram. Further work on this timing metric in CO-OFDM systems will be reported in a separate publication.

\section{B. Frequency Offset Synchronization}

The frequency offset in an OFDM system breaks the orthogonality between the subcarriers, incurring ICI penalty [28]. We envisage that for CO-OFDM systems, the frequency synchronization process is divided into two phases, frequency acquisition and tracking, which will be further discussed in Subsections 2.B.1 and 2.B.2.

\section{B.1. Frequency Acquisition}

Telecommunication lasers are usually locked to an International Telecommunication Union (ITU) frequency standard through a wavelength locker, but only with an accuracy of $\sim 2.5 \mathrm{GHz}$. This implies that the frequency offset could be anywhere from -5 to $5 \mathrm{GHz}$. Figure 5 shows the spectrum of the received CO-OFDM signal in relation to the local oscillator (LO) laser frequency. The excessive frequency offset $f_{\text {off }}$ brings two problems to the CO-OFDM system: (i) the highest RF frequency $\left(f_{\max }\right.$ in Fig. 5) of the RF OFDM signal after direct downconversion is increased by the amount of frequency

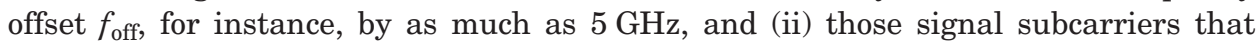
happen to overlap with the receiver LO will have degraded performance due to direct downconversion DC leakage. The former problem leads to excessive RF bandwidth expansion, therefore increasing the receiver cost. The latter problem may not be a severe issue for the point-to-point networks where the feedback to the transmitter is allowed such that the subcarriers with degraded performance will not be filled. But it will be quite challenging for an optical broadcast network where one CO-OFDM signal stream will be dropped at multiple points. The local receiver laser frequencies of multiple users are different if they are not frequency tracked to the transmit laser, and subsequently there are no common subcarriers that can be unfilled to accommodate the DC leakage problem. The purpose of the frequency acquisition is to first coarse estimate the frequency offset and bring the receiver laser approximately within several times of the subcarrier frequency spacing, typically approximately $100-500 \mathrm{MHz}$ from that of the transmit laser. Therefore we believe that coarse frequency (100-500 MHz accuracy) control is preferred in CO-OFDM systems in order to simplify the receiver signal processing. There are many frequency acquisition algorithms that have been proposed in the RF domain, such as the pilot-tone approach [31] or the shortened repeated DFT approach [32].

In CO-OFDM systems, since the initial frequency offset could be as much as several gigahertz, the initial acquisition can also be obtained by sending a long-stream $\mathrm{CW}$ signal (tens or hundreds of OFDM symbols) and measuring the frequency of the offset tone. The error signal is used to control the local laser to bring its frequency close to that of the transmit laser. After that, the second phase of frequency tracking could be initiated.

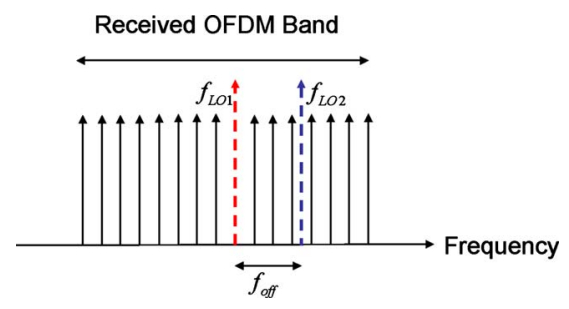

Fig. 5. Frequency spectrum of the received OFDM signal in relation to the local oscillator laser. $f_{L O 1}$ and $f_{L O 2}$ are, respectively, the transmitter and receiver laser frequencies. 


\section{B.2. Frequency Tracking}

The purpose of the frequency tracking is to identify the magnitude of the frequency offset and compensate for it. Again various approaches can be used for this purpose. We will use the Schmidl and Cox approach for the frequency tracking [29]. From Eq. (12), we arrive at

$$
R_{\hat{d}}=\sum_{m=1}^{N_{s c} / 2}\left|r_{m+\hat{d}}^{0}\right|^{2} e^{j\left(\pi f_{\text {off }} / \Delta f\right)}+o(n),
$$

where $o(n)$ stands for the residual term with the magnitude of the order of the noise component. It follows from Eq. (14) that the offset frequency can be estimated as

$$
\hat{f}_{\text {off }}=\frac{\Delta f}{\pi} \angle\left(R_{\hat{d}}\right),
$$

where $\hat{d}$ stands for the estimated DFT window timing and $\angle\left(R_{\hat{d}}\right)$ stands for the angle of the complex value of the correlation function of $R_{\hat{d}}$. The accuracy of Eq. (15) can be improved by sending multiple Schmidl and Cox [29] pilot symbols.

Once the frequency offset is estimated, the received sampled signal will be compensated as

$$
r_{c}(t)=\exp \left(-j 2 \pi \hat{f}_{\text {off }} t\right) r(t) .
$$

The frequency offset compensated signal $r_{c}(t)$ can be used for DFT to obtain the received information symbol $r_{k i}$ in Eq. (8), and subsequent subcarrier recovery for symbol decision is discussed in Subsection 2.C.

\section{C. Subcarrier Recovery: Channel Estimation and Phase Estimation}

From the channel model of Eq. (8), there are three factors that lead to the rotations of the receiver information symbol constellation for $r_{k i}$, and they are (i) the channel dispersion that gives frequency-selective dependence across the OFDM spectrum, (ii) the DFT sampling timing offset that generates a phase term linear with the subcarrier frequency, and (iii) the phase noises from the transmit and receive lasers. The time constants for the three factors are different. The first one changes on the time scale of a millisecond resulting from mechanical movement. In particular, the chromatic dispersion (CD) varies in response to the diurnal temperature fluctuation. The polarization-mode dispersion (PMD) varies due to the mechanical and temperature fluctuation on the time scale of a millisecond. The second one is caused by the sampling clock rate offset and may need to be reset from every microsecond to tens of microseconds. The third one comes from the laser phase noise with linewidth ranging from $100 \mathrm{KHz}$ to several megahertz, which needs to be tracked on a symbol-by-symbol basis. The factors (i) and (ii) are dealt with through channel estimation. The factor (iii) is treated through phase estimation and compensation.

We further assume that the signal processing is performed in blocks, each containing a large number of OFDM symbols. Within each block, the optical channel is assumed to be invariant, whereas the CPE varies on the OFDM symbol basis. Subsequently, the subcarrier recovery includes two baseband signal processings-channel and phase estimation. There are various methods of channel estimation such as the time-domain pilot-assisted and the frequency-domain assisted approaches [16,33,34]. We will focus on the carrier recovery based upon frequency-domain pilot carriers or pilot symbols. Figure 6 shows the two-dimensional time-frequency structure for one OFDM block, which includes $N_{s c}$ subcarriers in frequency and $N_{f}$ OFDM symbols in time. The preamble is added at the beginning to realize DFT window synchronization and channel estimation. The channel transfer function can be estimated as

$$
\hat{h}_{k}=\sum_{i=1}^{p} e^{-j<r_{k_{1} i} r_{k i} / c_{k i}},
$$

where $c_{k i}$ and $r_{k i}$ are, respectively, transmitted and received pilot subcarriers; $\angle r_{k_{1} i}$ is the angle for the $k_{1}$ th carrier (an arbitrary reference carrier) in the $i$ th OFDM symbol; and $p$ is the number of pilot symbols. The additional phase compensation of $-\angle r_{k_{1} i}$ is needed to remove the influence of the CPE. The accuracy of $\hat{h}_{k}$ can be further 


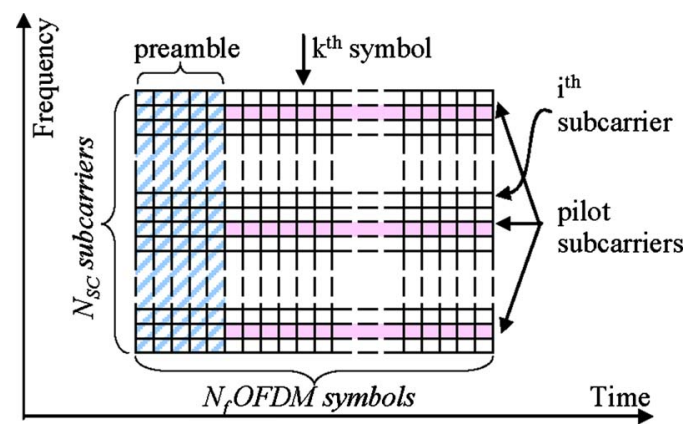

Fig. 6. Time and frequency representation of a block of CO-OFDM signal.

improved if the functional dependence of $\hat{h}_{k}$ on the subcarrier frequency is known. For instance, one pilot symbol could be sufficient for chromatic dispersion estimation if a quadratic function is used to interpolate the channel transfer function. The estimated channel transfer function has uncertainty of a common phase, which can be included in the CPE $\phi_{i}$. The phase estimation is to estimate the CPE $\phi_{i}$ due to the laser phase noise. We assume that the $N_{p}$ pilot subcarriers are used for phase estimation; thus the maximum likelihood $\mathrm{CPE} \phi_{i}$ can be estimated as

$$
\phi_{i}=\arg \left(\sum_{k=1}^{N_{p}} C_{k i}^{\prime} h_{k}^{*} C_{k i}^{*} / \delta_{k}^{2}\right),
$$

where $\delta_{k}$ is the standard deviation of the constellation spread for the $k$ th subcarrier. When $\delta_{k}$ is assumed to be constant across all the subcarriers, Eq. (18) will reduce to the least-squares (LS) method [35]. After the channel estimation [Eq. (17)] and the phase estimation [Eq. (18)], the subcarrier recovery can be completed using Eq. (9), in essence, to reconstruct the constellation for each subcarrier.

\section{Coherent Optical MIMO-OFDM Models}

In Section 2, the CO-OFDM signal is modeled as a one-element scalar. However, it is well known that single-mode fiber supports two modes in the polarization domain. Each polarization mode travels with a slightly different speed due to PMD and undergoes different loss due to polarization-dependent loss (PDL). Therefore the complete CO-OFDM model requires the mathematical description of the two-polarization coupling including these polarization effects as well as the fiber chromatic dispersion. As shown in [13], such a model in essence is a $2 \times 2$ MIMO-OFDM model. The timedomain signal [13] at the transmit is given by

$$
\begin{gathered}
\vec{s}(t)=\sum_{i=-\infty}^{+\infty} \sum_{k=-(1 / 2) N_{s c}+1}^{(1 / 2) N_{s c}} \vec{c}_{i k} \Pi\left(t-i T_{s}\right) \exp \left(j 2 \pi f_{k}\left(t-i T_{s}\right)\right), \\
\vec{s}(t)=\left(\begin{array}{l}
s_{x} \\
s_{y}
\end{array}\right), \quad \vec{c}_{i k}=\left(\begin{array}{c}
c_{i k}^{x} \\
c_{i k}^{y}
\end{array}\right), \\
f_{k}=\frac{k-1}{t_{s}}, \\
\Pi(t)= \begin{cases}1 & \left(-\Delta_{G}<t \leq t_{s}\right) \\
0 & \left(t \leq-\Delta_{G}, t>t_{s}\right)\end{cases}
\end{gathered}
$$

where $s_{x}$ and $s_{y}$ are the two polarization components for $\vec{s}(t)$ in the time domain; $\vec{c}_{i k}$ is the transmitted OFDM information symbol in the form of Jones vector for the $k$ th subcarrier in the $i$ th OFDM symbol; $c_{i k}^{x}$ and $c_{i k}^{y}$ are the two polarization components for $\vec{c}_{i k} ; f_{k}$ is the frequency for the $k$ th subcarrier; $N_{s c}$ is the number of OFDM subcarriers; and $T_{s}, \Delta_{G}$, and $t_{s}$ are the OFDM symbol period, guard interval length, and observation period, respectively. The Jones vector $\vec{c}_{i k}$ is employed to describe the generic OFDM information symbol regardless of any polarization configuration for the 


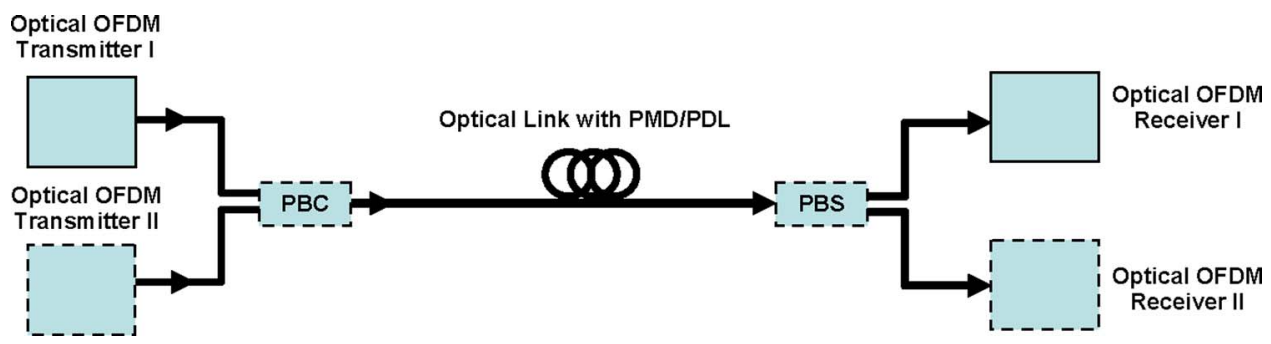

Fig. 7. Conceptual diagram of a $2 \times 2$ coherent optical MIMO-OFDM model. The dashed line on the devices indicates the variations of the architecture with the option to remove the device from the configuration. PBC, polarization beam combiner; PBS, polarization beam splitter.

OFDM transmitter. In particular, the $\vec{c}_{i k}$ encompasses various modes of the polarization generation including single polarization, polarization multiplexing, and polarization modulation, as they all can be represented by a two-element Jones vector $\vec{c}_{i k}$. The different scheme of polarization modulation for the transmitted information symbol is automatically dealt with during the initialization phase of OFDM signal processing by sending known training symbols. The received information symbol after proper DFT window and frequency offset synchronization is given by

$$
\begin{gathered}
\vec{c}_{k i}^{\prime}=e^{j \phi_{i}} e^{j \Phi_{D}\left(f_{k}\right)} T_{k} \vec{c}_{k i}+\vec{n}_{k i}, \\
T_{k}=\prod_{l=1}^{N} \exp \left(\left(-\frac{1}{2} j \vec{\beta}_{l} f_{k}-\frac{1}{2} \vec{\alpha}_{l}\right) \vec{\sigma}\right), \\
\Phi_{D}\left(f_{k}\right)=\pi c D_{t} f_{k}^{2} / f_{L D 1}^{2},
\end{gathered}
$$

where $\vec{c}_{k i}^{\prime}=\left(\begin{array}{ll}c_{x}^{\prime k i} & c_{y}^{\prime k i}\end{array}\right)^{t}$ is the received information symbol in the form of the Jones vector for the $k$ th subcarrier in the $i$ th OFDM symbol, $\vec{n}_{k i}=\left(\begin{array}{cc}n_{x}^{k i} & n_{y}^{k i}\end{array}\right)^{t}$ is the noise including two polarization components, $T_{k}$ is the Jones matrix for the fiber link, $N$ is the number of PMD-PDL cascading elements represented by their birefringence vector $\vec{\beta}_{l}$ and PDL vector $\vec{\alpha}_{l}$ [36], $\vec{\sigma}$ is the Pauli matrix vector [36], $\Phi_{D}\left(f_{k}\right)$ is the phase dispersion owing to the fiber CD [1], and $\phi_{i}$ is again the CPE noise owing to the phase noises from the lasers and RF LO at both the transmitter and receiver [1].

Equations (23)-(25) show a $2 \times 2$ coherent optical MIMO-OFDM (CO-MIMOOFDM) model relating the two outputs $c_{i k}^{\prime}{ }^{x}$ and $c_{i k}^{\prime}{ }^{y}$ to the two inputs $c_{i k}^{x}$ and $c_{i k}^{y}$, with the physical representation shown in Fig. 7. Such a MIMO model has been widely investigated to improve the performance of wireless systems [37]. We also note that an optical MIMO model has been proposed and analyzed in single carrier systems [38].

There are three other variations of CO-MIMO-OFDM models, namely, $1 \times 1$ singleinput-single-output (SISO)-OFDM, $2 \times 1$ two-input-single-output (TISO)-OFDM, and $1 \times 2$ single-input-two-output (SITO)-OFDM [13]. Except for SISO-OFDM, all MIMOOFDM variations can be PMD resilient and support coherent detection without optical polarization tracking. In Section 4, we will present in detail the simulation and experimental work on the variations of MIMO-OFDM configurations.

\section{Simulation and Experimental Study of MIMO-OFDM Systems}

The first attempt to take advantage of the OFDM to combat PMD is reported in [2], exploiting polarization receiver diversity, which in essence is a $1 \times 2$ MIMO-OFDM system. It shows that the PMD in the installed fiber network can be gracefully mitigated without an optical compensator. It is further suggested that it may be advantageous to introduce PMD in the systems to assist the polarization diversity, in order to mitigate PDL impairment [39]. This may present an intriguing paradigm shift in studying PMD effects, as before that work [39], the PMD had always been considered an impairment that should be removed from the fiber as much as possible. The first experiment with a $1 \times 2$ MIMO-OFDM in the polarization domain was reported in [13], where a CO-OFDM signal at $10.7 \mathrm{Gbits} / \mathrm{s}$ was successfully recovered after $900 \mathrm{ps}$ differential group delay (DGD) and $1000 \mathrm{~km}$ transmission through standard single- 
mode fiber (SSMF) without optical dispersion compensation. A $2 \times 2$ MIMO-OFDM using direct up-downconversion is reported for $1000 \mathrm{~km}$ SSMF transmission at $21.4 \mathrm{Gbits} / \mathrm{s}$ [40] and using intermediate frequency up-downconversion is reported for $4160 \mathrm{~km}$ transmission of 16 channels at $52.5 \mathrm{Gbits} / \mathrm{s}$ [14]. We note that PMD mitigation using direct-detection optical OFDM has also been proposed and investigated $[27,41,42]$. In the remainder of this section, we will present these important results of coherent optical MIMO-OFDM through simulation as well as experimental work.

\section{A. Polarization-Mode Dispersion: Detriment or Benefit?}

In this section, we will review the interaction of PMD and PDL in a $1 \times 2$ MIMOOFDM system. The system parameters for the simulation can be found in [39]. In a polarization-impaired system, we use outage probability to characterize the system performance, which is defined as the probability that the $\mathrm{Q}$ penalty exceeds a certain value. Figure 8 shows the outage probability as a function of the $Q$ penalty. A mean PDL of $5 \mathrm{~dB}$ is assumed for all the simulations. We can see that without PMD in the system, there is a system $Q$ penalty of $4.4 \mathrm{~dB}$ at the outage probability of $10^{-3}$. However, if we introduce a mean PMD of $150 \mathrm{ps}$ into the otherwise same system, to maintain the same outage probability of $10^{-3}$, the system penalty is reduced to $3.0 \mathrm{~dB}$ for a $10 \mathrm{Gbit} / \mathrm{s}$ system, which is a $1.4 \mathrm{~dB}$ improvement over the case of no PMD in the fiber link. A higher bit rate system at $40 \mathrm{Gbits} / \mathrm{s}$ further reduces the $\mathrm{Q}$ penalty to $2.4 \mathrm{~dB}$ with a PMD of $150 \mathrm{ps}$, a $2 \mathrm{~dB}$ improvement over the case of no PMD. This further improvement is attributed to a higher degree of diversity with a larger number of subcarriers for a higher bit rate system. We can see that the PMD in the fiber improves the system margin for PDL-induced penalty, and the improvement is enhanced for a higher bit rate system. We also note that a relatively large PMD is needed of the order of $150 \mathrm{ps}$ to gain the benefit, which usually is not available in the fiber link. One of the possibilities is to artificially introduce high PMD into the fiber or optical components. Mitigation of a large PDL in CO-OFDM systems may significantly loosen the PDL specification for various components and subsequently bring appreciable cost savings. Finally, the fiber nonlinearity, including self-phase modulation (SPM) and cross-phase modulation (XPM), will be reduced in PMD-supported CO-OFDM systems as previously reported for single-carrier systems [43]. Equivalently, the interchannel and intrachannel OFDM subcarrier four-wave mixing (FWM) can be greatly reduced by a large phase mismatch between subcarriers in the presence of a large PMD.

The results can be better understood by drawing a comparison with the RF MIMOOFDM systems [37]. It is well-known that the performance of MIMO-OFDM in the space domain can be improved through introduction of the delay spread into the system [37]. This is because it will bring frequency selectivity into the channel. Similarly, introducing the PMD into the MIMO-OFDM channel in the polarization domain will bring frequency selectivity into the optical channel, and subsequently the performance is expected to improve. Although whether any PMD and how much PMD should be reintroduced into the fiber will be influenced by other factors, the work presented here adopts a fresh angle to explore this old PMD problem. Moreover, this work may be extremely significant and timely to those carriers who hold a significant amount of old fiber with large PMD and still wish to upgrade to $40 \mathrm{Gbit} / \mathrm{s}$ systems and beyond. Our findings show these fibers may not be necessarily bad for future high-speed transmission systems.

4.B. $1 \times 2$ MIMO-OFDM Experiment: Polarization-Diversity Detection

This Subsection and Subsections 4.C and 4.D encompass three variations of optical MIMO-OFDM systems, each organized to comprise complete experimental setup,

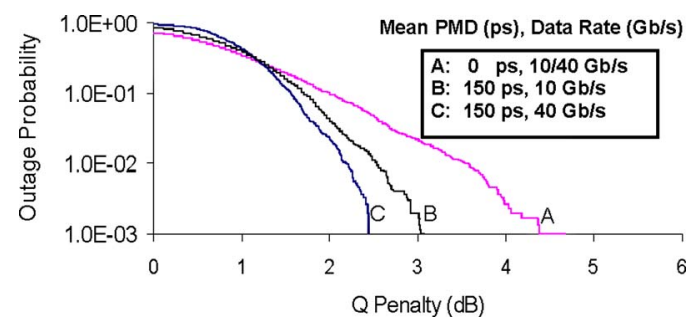

Fig. 8. Outage probability as a function of $\mathrm{Q}$ penalty with varying $\mathrm{PMD}$ and data rate. 
OFDM parameter description, experimental results, and associated discussion, which can be read independently without referring to other sections.

Figure 9 shows the experimental setup for verifying the PMD-supported CO-OFDM systems using $1 \times 2 \mathrm{MIMO}-\mathrm{OFDM}$ architecture. The OFDM signal is generated by using a Tektronix arbitrary waveform generator (AWG) as a RF OFDM transmitter. The time domain waveform is first generated with a Matlab program including mapping $2^{15}-1$ pseudorandom binary sequences (PRBSs) into 77 corresponding subcarriers with quadrature phase-shift keying (QPSK) encoding within multiple OFDM symbols, which are subsequently converted into time domain using inverse fast Fourier transform (IFFT) and inserted with guard interval (GI). The number of OFDM subcarriers is 128 and the GI is 1/8 of the observation period. Only the middle 87 subcarriers out of 128 are filled, from which 10 pilot subcarriers are used for phase estimation. This filling is to achieve tighter spectral control by oversampling and should not be confused with the selective carrier filling due to channel fading. The BER performance is measured using all 77 data bearing channels. The OFDM digital waveform of $s_{B}(t)$ [Eq. (1)] is of complex value. Its real and imaginary parts are uploaded into the AWG operated at $10 \mathrm{GS} / \mathrm{s}$, and two-channel analog signals each representing the real and imaginary components of the complex OFDM signal are generated synchronously. The so-generated OFDM waveform carries $10.7 \mathrm{Gbit} / \mathrm{s}$ data. These two signals are fed into I and $\mathrm{Q}$ ports of an optical I/Q modulator, respectively, to perform direct upconversion of OFDM baseband signals from the RF domain to the optical domain [25]. The optical OFDM signal from the I/Q modulator is first inserted into a homemade PMD emulator and then fed into a recirculation loop, which includes one span of $100 \mathrm{~km}$ SSMF fiber and an EDFA to compensate the loss. The advantages of such a direct upconversion scheme are (i) the required electrical bandwidth is less than half that of IF counterpart and (ii) there is no need for an image-rejection optical filter [25]. The launch power into each fiber span is set at $-8 \mathrm{dBm}$ to avoid the nonlinearity, and the received OSNR is $14 \mathrm{~dB}$ after $1000 \mathrm{~km}$ transmission. At the receive end, the polarization-diversity detection is employed. We use IF downconversion where the LO laser frequency is offset with that of the signal laser [3]. The output optical signal from the loop is first split into two polarizations, each fed into an OFDM OTR downconverter that includes a balanced receiver and a local laser. The two RF signals for the two polarizations are then input into a Tektronix time-domain-sampling (TDS) scope and acquired synchronously. The RF signal traces corresponding to the $1000 \mathrm{~km}$ transmission are acquired at $20 \mathrm{GS} / \mathrm{s}$ and processed with a Matlab program as a $\mathrm{RF}$ OFDM receiver. The RF OFDM receiver signal processing involves (1) fast Fourier transform (FFT) window synchronization using the format of Schmidl and Cox [29] to identify the start of the OFDM symbol, (2) software downconversion of the OFDM RF signal to baseband by a complex pilot subcarrier tone, (3) phase estimation for each OFDM symbol, (4) channel estimation in terms of a Jones vector and Jones matrix, and (5) constellation construction for each carrier and BER computation.

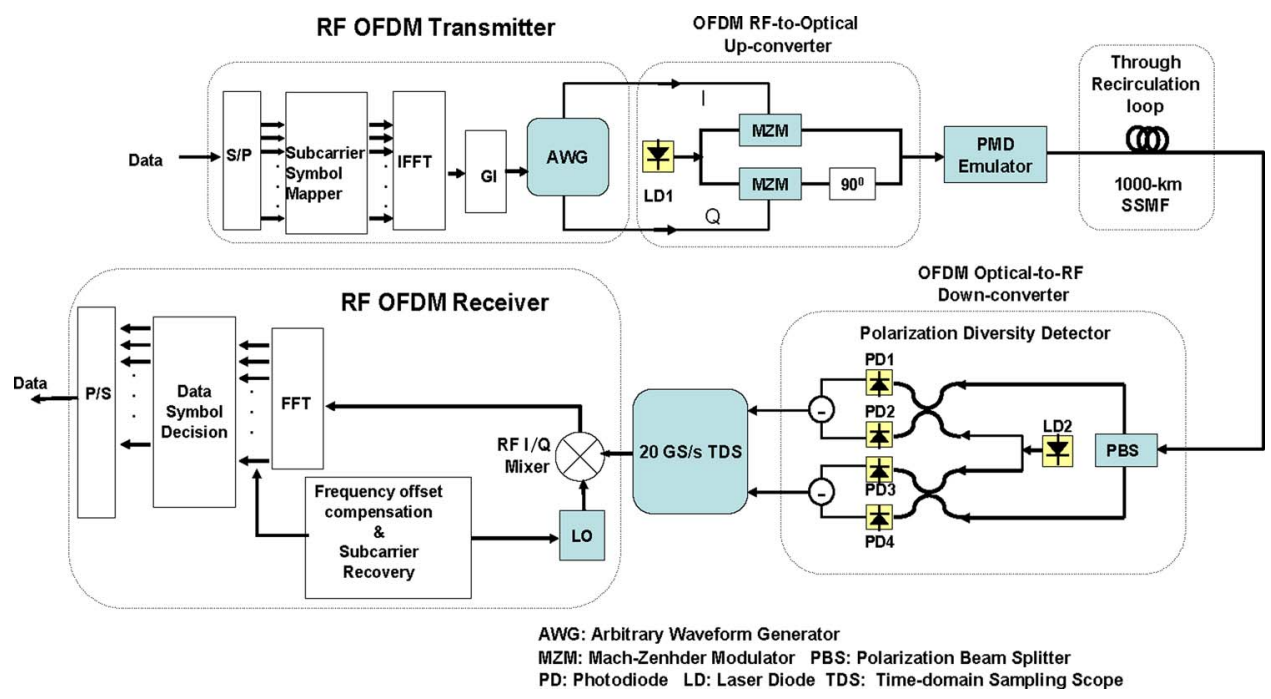

Fig. 9. Experimental setup for a $1 \times 2$ MIMO-OFDM in the polarization domain. 


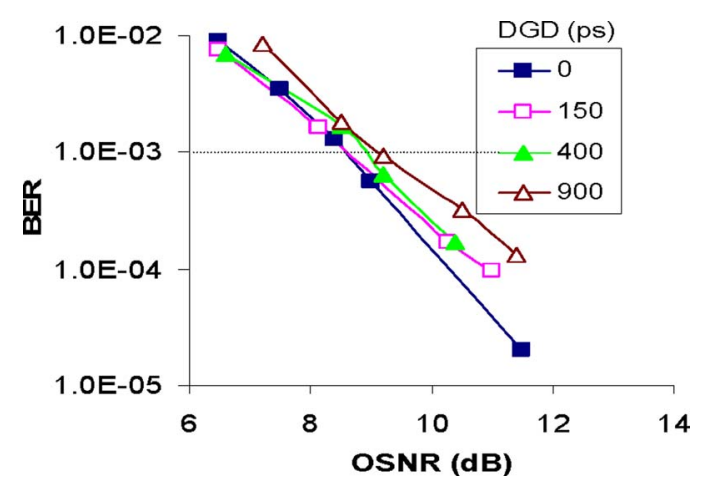

Fig. 10. BER performance of a CO-OFDM signal.

The RF OFDM signal is downconverted to baseband by simply multiplying a complex residual carrier tone in software, eliminating the need for a hardware RF LO. This complex carrier tone can be supplied with the pilot symbols or pilot subcarriers as discussed in Section 2. The downconverted baseband signal is segmented into blocks of 400 OFDM symbols with the cyclic prefix removed, and the individual subcarrier symbol in each OFDM symbol is recovered by using FFT.

The associated channel model after removing the phase noise $\phi_{i}$ is given by [39]

$$
\vec{c}_{k i}^{\prime p}=H_{k} c_{k i}+\vec{n}_{k i}^{p},
$$

where $\vec{c}_{k i}^{\prime p}$ is the received OFDM information symbol in a Jones vector for the $k$ th subcarrier in the $i$ th OFDM symbol, with the phase noise removed; $H_{k}=e^{j \Phi_{D}\left(f_{k}\right)} T_{k}$ is the channel transfer function; and $\vec{n}_{k i}^{p}$ is the random noise.

The expectation values for the received phase-corrected information symbols $\vec{c}_{k i}^{\prime p}$ are obtained by averaging over a running window of 400 OFDM symbols. The expectation values for four QPSK symbols are computed separately by using received symbols $\vec{c}_{k i}^{\prime}$. An error occurs when a transmitted QPSK symbol in a particular subcarrier is closer to the incorrect expectation values at the receiver. The process described here is the same as finding the channel transfer function $H_{k}$ first and using Eq. (9) for transmitted symbol estimation and decision.

Figure 10 shows the BER performance of the CO-OFDM signal after 900 ps DGD and $1000 \mathrm{~km}$ SSMF transmission. The optical power is evenly launched into the two principal states of the PMD emulator. The measurements using other launch angles show insignificant difference. Compared with the back-to-back case, it has less than $0.5 \mathrm{~dB}$ penalty at the BER of $10^{-3}$. The magnitude of the PMD tolerance is shown to be independent of the data rate [2]. Therefore we expect that the same PMD resilience in absolute magnitude will hold for $40 \mathrm{Gbits} / \mathrm{s}$ if faster ADC-DACs are available at $20 \mathrm{GS} / \mathrm{s}$ and above.

\section{C. $2 \times 1$ MIMO-OFDM Experiment: Polarization-Time Coding for Optical Broadcast} Networks

As we discussed in Section 3, the CO-OFDM systems are in essence a $2 \times 2$ MIMOOFDM channel. Consequently, it seems to be prohibitively expensive for an access network. However, the complexity of the receiver structure can be significantly reduced by employing transmit diversity. This is analogous to the wireless network where multiple antennas in the base station are used, the system performance is greatly enhanced without significantly increasing the cost of the mobile user. In the transmit diversity scheme for CO-OFDM, namely, for a $2 \times 1$ MIMO-OFDM system, two OFDM transmitters are used, one for each polarization. We send two consecutive OFDM symbols with orthogonal polarization. By processing the OFDM received signals properly, both polarization signals can be recovered, without resorting to a polarization-diversity receiver. Mathematically, the two consecutive OFDM symbols with orthogonal polarization in the form of Jones vector are given by

$$
\vec{c}_{1}=\left(c_{1 x}, c_{1 y}\right)^{t}, \quad \vec{c}_{2}=\left(-c_{1 y}^{*}, c_{1 x}^{*}\right)^{t} .
$$

Assume that the fiber transmission Jones Matrix $H$ is 


$$
H=\left(\begin{array}{ll}
h_{x x} & h_{x y} \\
h_{y x} & h_{y y}
\end{array}\right) .
$$

To simplify the receiver architecture, only one polarization of the received signal, along the polarization of the local laser, is detected in the receiver. Without loss of generality, the $x$ polarization component is assumed to be detected. The two received scalar OFDM symbols $c_{1}^{\prime}$ and $c_{2}^{\prime}$ corresponding to transmitted $\vec{c}_{1}$ and $\vec{c}_{2}$ in the $x$ polarization are, respectively,

$$
c_{1}^{\prime}=h_{x x} c_{1 x}+h_{x y} c_{1 y}, \quad c_{2}^{\prime}=-h_{x x} c_{1 y}^{*}+h_{x y} c_{1 x}^{*} .
$$

Solving Eq. (29), we can recover the $\vec{c}_{1}$ as

$$
\vec{c}_{1}=H^{\prime}\left(\begin{array}{c}
c_{1}^{\prime} \\
c_{2}^{\prime *}
\end{array}\right), \quad H^{\prime}=\left(\begin{array}{cc}
h_{x x} & h_{x y} \\
h_{x y}^{*} & -h_{x x}^{*}
\end{array}\right)^{-1} .
$$

It can be seen from Eq. (30), even though one polarization is detected, that, by using orthogonal polarization for two consecutive symbols, the two transmitted polarization symbols $\left(c_{1 x}\right.$ and $c_{1 y}$ ) can be recovered. Because of the complementarity of $h_{x x}$ and $h_{x y}$, the performance is independent of the polarization rotation and the PMD in the fiber. This polarization-time coding is equivalent to space-time coding in wireless systems [44]. This polarization-time coding scheme has been proposed and analyzed in single-carrier systems [45].

OFDM is inherently spectrally efficient due to the spectral overlapping between subcarriers, allowing for approximately $6 \mathrm{GHz}$ optical spectrum for $10 \mathrm{Gbit} / \mathrm{s}$ data using QPSK modulation [13]. By using direct up-downconversion architecture, where the transmitter and receiver lasers are placed at the center frequency of the optical OFDM spectrum, the electrical bandwidth required for both the transmitter and receiver is only half the optical spectrum bandwidth, which is $3 \mathrm{GHz}$ for a $10 \mathrm{Gbit} / \mathrm{s}$ system. This is fundamentally different from other modulation approaches where the electrical bandwidth can be reduced through excessive electrical filtering or through higher-order modulation, which all will inevitably result in system penalty. This signifies that the $10 \mathrm{Gbit} / \mathrm{s}$ system can potentially use cheap $2.5 \mathrm{GHz}$ components. Additionally, the receiver sensitivity will be greatly enhanced through coherent detection, which has been a known fact for more than a decade. Considering all the advantages, we conclude that CO-OFDM may have a significant role for downstream systems in a high-speed and long-reach passive optical network (PON) beyond 10-100 Gbits/s, where the dispersion and receiver sensitivity are of critical importance [46].

Figure 11 shows the experimental setup for verifying the transmitter diversity scheme for a broadcast network. The OFDM signal is generated by using a Tektronix AWG as a RF OFDM transmitter. The time domain waveform is first generated with a Matlab program including mapping $2^{15}-1$ PRBS into 77 corresponding subcarriers with QPSK encoding within multiple OFDM symbols, which are subsequently converted into the time domain using IFFT and inserted with a GI. The number of OFDM subcarriers is 128 and the GI is $1 / 8$ of the observation period. Only the middle 87 subcarriers out of 128 are filled, of which 10 pilot subcarriers are used for phase estimation. The real and imaginary parts of the OFDM waveforms are uploaded into the AWG operated at $10 \mathrm{GS} / \mathrm{s}$, and two channel analog signals each representing the real and imaginary components of the complex OFMD signal are generated synchro-

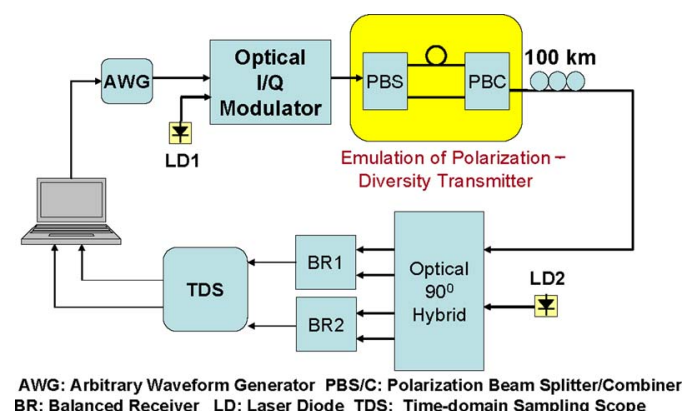

Fig. 11. Experimental setup for a polarization-diversity transmitter scheme for a broadcast network. 


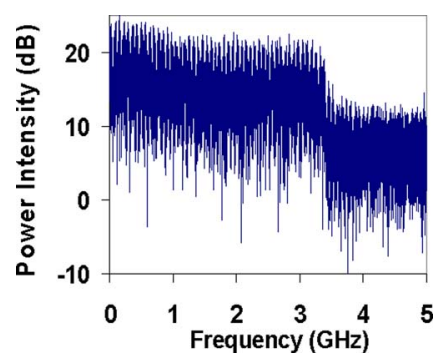

Fig. 12. Electrical spectrum for the $10 \mathrm{Gbit} / \mathrm{s}$ signal.

nously. The so-generated OFDM waveform carries $10.7 \mathrm{Gbit} / \mathrm{s}$ data. These two signals are fed into the I and $\mathrm{Q}$ ports of an optical I/Q modulator, respectively, to perform direct upconversion of OFDM baseband signals from the RF domain to the optical domain. The polarization transmit diversity is emulated by using a polarization beam splitter (PBS) to generate two OFDM streams. The two polarization streams are delayed with respect to each other by an OFDM symbol period of $14.4 \mathrm{~ns}$ and then combined into one optical signal. We use one span of $100 \mathrm{~km}$ to emulate the fiber plant. At the receive end, OTR direct downconversion is performed. In particular, the LO laser center frequency is tuned approximately to that of the incoming signal, and both the signal and LO are fed into an optical $90^{\circ}$ hybrid. The I/Q optical signals are then input into two balanced detectors. A single detector has been shown to have almost the same performance [10]. The two RF signals from the photodetectors are then input into a Tektronix TDS scope and are acquired at $20 \mathrm{GS} / \mathrm{s}$ and processed with a Matlab program. No phase-locking and polarization control between the LO and signal are needed. The RF OFDM receiver signal processing again involves (i) FFT window synchronization using the format of Schmidl and Cox [29] to identify the start of the OFDM symbol, (ii) software downconversion of the OFDM RF signal to baseband to remove residual frequency offset, (iii) phase estimation and channel estimation, and (iv) constellation construction for each carrier and BER computation. The channel matrix $H$ is estimated by sending 50 OFDM symbols. The OFDM symbols are partitioned into four groups with proper coding similar to Eq. (27), resulting in two of the four OFDM symbols with orthogonal polarizations, which are subsequently measured for system performance.

Figure 12 shows the electrical spectrum for the received $10 \mathrm{Gbit} / \mathrm{s}$ signal. The OFDM spectrum is tightly bounded within $3 \mathrm{GHz}$. More importantly, although our system shows $3 \mathrm{~dB}$ roll-off at $3 \mathrm{GHz}$, it does not seem to affect the performance, as the OFDM spectrum is limited to only $3 \mathrm{GHz}$. Such a high spectral efficiency will prove critical for future high-speed access networks. Figure 13 shows receiver sensitivity without an optical amplifier after $100 \mathrm{~km}$ transmission at $10 \mathrm{Gbits} / \mathrm{s}$. It is expected that the OFDM signal may have an error floor of $10^{-6}$ because of intentional clipping. Subsequently, error correction is required for OFDM systems. We perform measurement at the BER of $10^{-1}$ to $10^{-4}$. The lower end of the BER is limited by the number of OFDM symbols processed (approximately 500 each time). We do not see much penalty after $100 \mathrm{~km}$ transmission. This is expected as much longer transmission has been demonstrated without optical dispersion compensation but with a more sophisticated polarization-diversity receiver $[13,14]$. This implies that CO-OFDM could enable much longer reach. We also note that in the experiment, the polarization in the link is free running and the system is immune to the polarization rotation in the link due to the polarization-diversity transmitter.

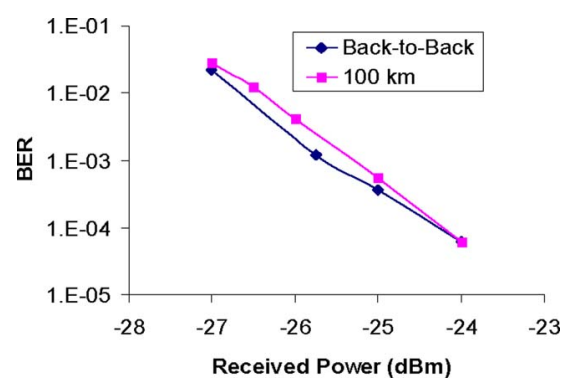

Fig. 13. Receiver sensitivity. 


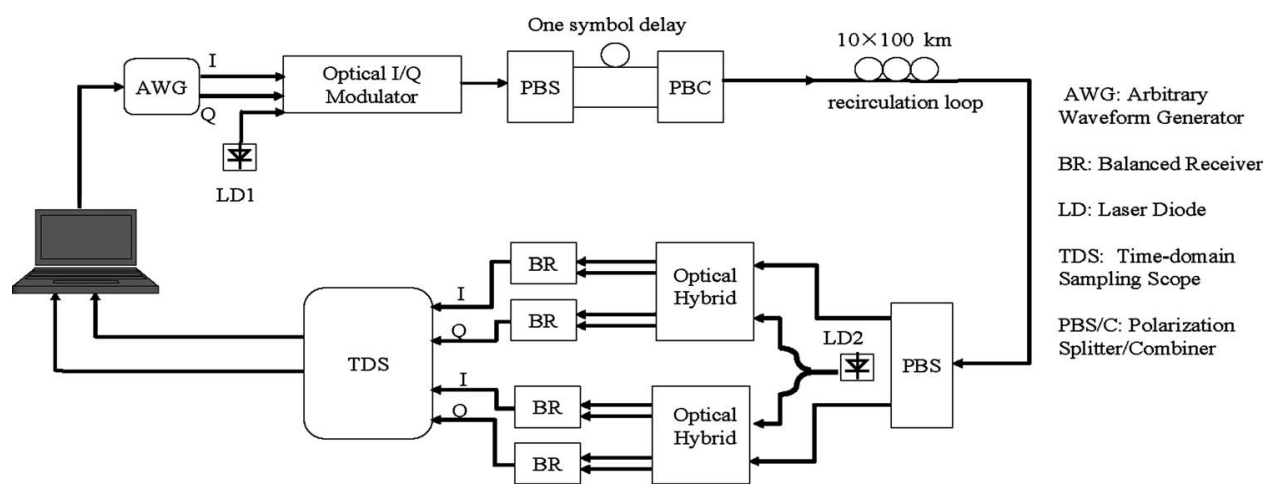

Fig. 14. Experimental setup for a $2 \times 2$ coherent optical MIMO-OFDM.

\section{D. $2 \times 2$ MIMO-OFDM in the Polarization Domain}

Two polarization modes supported in the fiber present an attractive avenue to double the fiber capacity. In this subsection, we will show the $2 \times 2$ MIMO-OFDM transmission that achieves the doubling of the system capacity without sacrificing the receiver sensitivity. Figure 14 shows the $2 \times 2$ coherent optical MIMO-OFDM (CO-MIMOOFDM) system. The time-domain digital waveform is first generated with a Matlab program including mapping $2^{15}-1$ PRBS into 87 corresponding subcarriers with QPSK encoding within multiple OFDM symbols, which are subsequently converted into the time domain using IFFT and inserted with a GI. The OFDM symbol period is $12.8 \mathrm{~ns}$ and the GI is $1.6 \mathrm{~ns}$. The digital waveform is expressed by a complex value, and its real and imaginary parts are uploaded into a Tektronix AWG operated at $10 \mathrm{GS} / \mathrm{s}$ per channel. Oversampling is done by filling 87 subcarriers out of 128 . Of them, 10 are reserved for pilot subcarriers. Each signal processing includes at least 500 OFDM symbols. An optical I/Q modulator is used for direct upconversion. The two transmitters are emulated as follows: The single-polarization optical OFDM signal at the output of the $\mathrm{I} / \mathrm{Q}$ modulator is first evenly split into two polarization branches with a PBS, with one branch delayed by one OFDM symbol period, i.e., $14.4 \mathrm{~ns}$ in this experiment. The two polarization branches are subsequently combined, emulating two independent transmitters, one on each polarization, resulting in a composite data rate of $21.4 \mathrm{Gbits} / \mathrm{s}$. The signal is then launched into a recirculation loop, which includes one span of $100 \mathrm{~km}$ SSMF and an EDFA to compensate the loss. The optical signal from the loop is tapped off and fed into a PBS for the polarizationdiversity coherent receiver. Each branch of the splitter is mixed with a local laser with an optical $90^{\circ}$ hybrid, and the I and Q ports from the optical $90^{\circ}$ hybrid are used for direct downconversion. Both transmit and receive lasers in this experiment are external-cavity lasers and have a laser linewidth of approximately $100 \mathrm{kHz}$. The frequency offset of the two lasers is set to near DC. The baseband signal from the balanced receivers is then fed into a Tektronix TDS scope operated at $20 \mathrm{GS} / \mathrm{s}$. The data trace recorded in a TDS scope is loaded into a computer and processed with a Matlab program as an OFDM receiver.

Figure 15(a) shows the back-to-back BER performance for $10.7 \mathrm{Gbit} / \mathrm{s}$ singlepolarization OFDM and $21.4 \mathrm{Gbit} / \mathrm{s} 2 \times 2 \mathrm{MIMO}-\mathrm{OFDM}$. Both cases are demonstrated

(a)

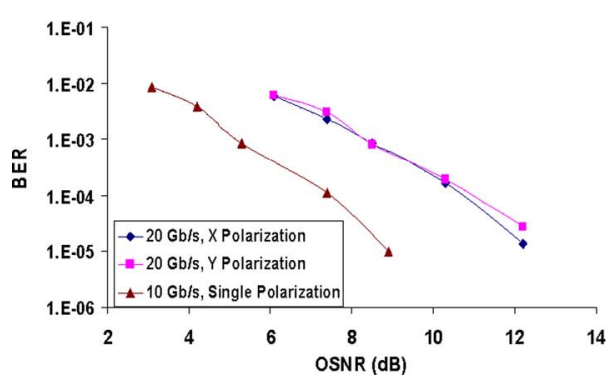

(b)

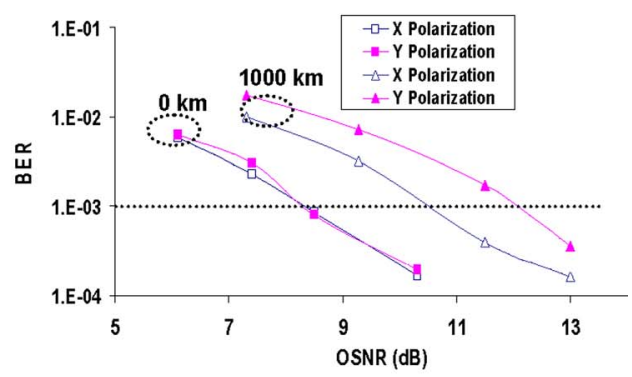

Fig. 15. (a) Back-to-back BER curve for 10.7 and $21.4 \mathrm{Gbit} / \mathrm{s}$ signals; (b) BER sensitivity for $2 \times 2$ MIMO OFDM back-to-back and $1000 \mathrm{~km}$ transmission. 
with direct up-downconversion. We can see that $21.4 \mathrm{Gbit} / \mathrm{s} 2 \times 2 \mathrm{MIMO}-\mathrm{OFDM}$ introduces $\sim 3 \mathrm{~dB}$ penalty compared with $10.7 \mathrm{Gbit} / \mathrm{s}$ single polarization. This is because the data rate for MIMO-OFDM is doubled, without increasing the required electrical bandwidth. Figure 15(b) shows the performance of a $21.4 \mathrm{Gbit} / \mathrm{s}$ MIMO-OFDM signal after $1000 \mathrm{~km}$ transmission. The launch power into the loop is selected to be $-5 \mathrm{dBm}$. It is observed that the two polarizations have almost the same performance at backto-back transmission while their performance has a difference of $1 \mathrm{~dB}$ at $1000 \mathrm{~km}$ transmission. This mismatch may be caused by imperfect channel estimation.

\section{Single-Carrier or Multicarrier: An Optical Debate}

We have so far limited our discussion to multicarrier frequency-domain equalization signal processing, which can be generalized to be one method of FDE. Although OFDM has gained popularity in the past decade and has been widely implemented in numerous communication standards, there has been on-going debate about which is superior, OFDM and SCFDE $[47,48]$. OFDM has two fundamental problems, which have been disapproved by the SCFDE camp, which are (i) large peak-to-averagepower ratio (PAPR) and (ii) sensitivity to phase noise. The SCFDE has to a large extent avoided these two problems. We would like to point out that the SCFDE here should not be confused with single-carrier time-domain equalization (SCTDE) based on a finite-impulse-response (FIR) filter [10-12]. As we will illustrate below, SCFDE more resembles OFDM in signal framing structure and signal processing that heavily relies on FFT-IFFT. We anticipate that a similar debate of "OFDM or SCFDE?" will spread to the optical domain.

In this section, we first describe in detail the configurations of CO-SCFDE and IO-SCFDE. We use incoherent here to refer to optical direct detection. In spite of higher complexity, CO-SCFDE has advantages over IO-SCFDE in spectral efficiency, PMD resilience, and OSNR sensitivity. Thus we focus our attention on CO-SCFDE and present the first experiment of CO-SCFDE transmission over $1000 \mathrm{~km}$ at $9.4 \mathrm{Gbits} / \mathrm{s}$.

Similar to the CO-OFDM system, a generic optical SCFDE (O-SCFDE) has five functional blocks as shown in Fig. 16. The major differences between O-SCFDE and O-OFDM systems are twofold: (i) the IDFT for SCFDE is located in the receiver instead of the transmitter and (ii) the symbol decision for SCFDE is made in the time domain instead of the frequency domain. Other than that, the O-SCFDE and O-OFDM look similar in terms of frame structure and signal equalization. However, because IDFT is performed in the receiver, the PAPR due to the IDFT is avoided at least at the start of the optical link. Second, since the decision is made for each bit in the time domain, the phase noise requirement of CO-SCFDE is less stringent than that of CO-OFDM.

Figure 17 shows the spectral allocation for IO-SCFDE and CO-SCFDE. In IO-SCFDE systems, the main optical carrier is offset with the main QPSK spectrum [Fig. 17(a)]. The QPSK spectrum may need spectral shaping so that its spectrum is narrowing up. The direct-detected signal gives the QPSK RF spectrum at IF [Fig. 17(b)]. In contrast, in CO-SCFDE systems, only the QPSK optical spectrum needs to be transmitted [Fig. 17(c)] with the main subcarrier completely suppressed. Figure 17(d) shows the coherently detected signal spectrum for either the I or Q component. It can be seen that CO-SCFDE has better optical and electrical spectral efficiency than IO-SCFDE. Additionally, CO-SCFDE is superior in OSNR sensitivity due to coherent detection. Similar to CO-OFDM, CO-SCFDE with a polarization-diversity receiver leads to $\mathrm{PMD}$ and polarization rotation-resilient transmission. Therefore we limit the discussion to the coherent flavor of SCFDE.

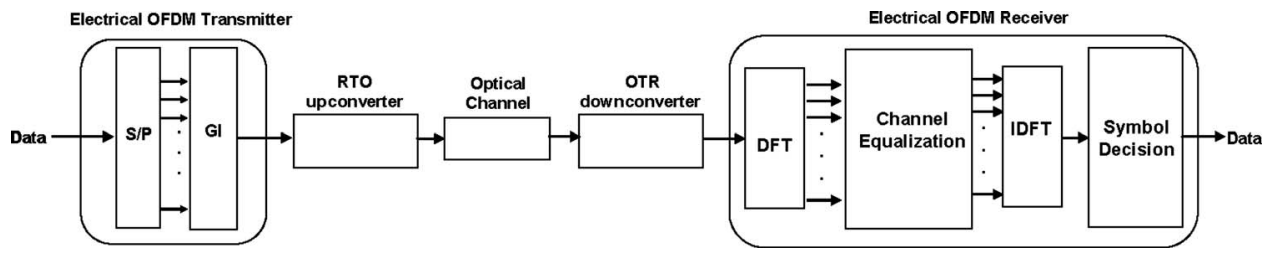

Fig. 16. Conceptual diagram of an optical SCFDE (O-SCFDE) system. 
(a)

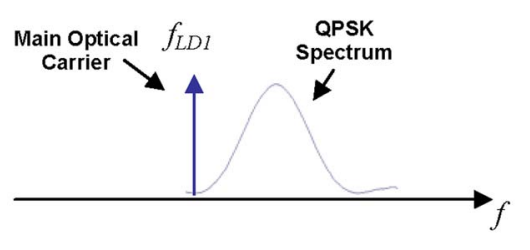

(c)

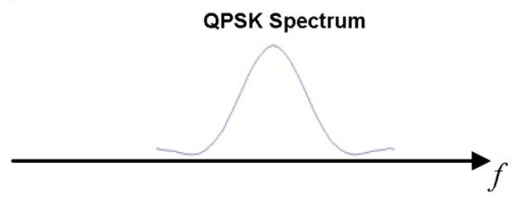

(b)

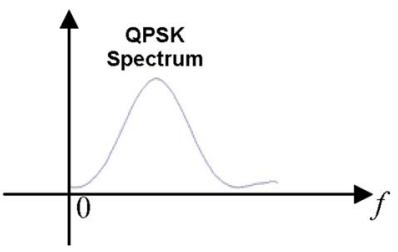

(d)

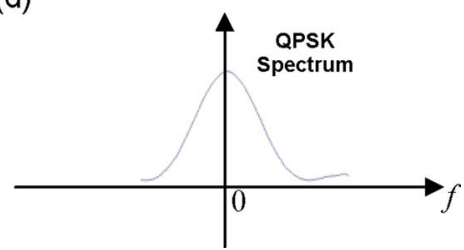

Fig. 17. (a) IO-SCFDE optical spectrum, (b) IO-SCFDE electrical spectrum, (c) CO-SCFDE optical spectrum, and (d) CO-SCFDE electrical spectrum for the I or Q component. In CO-SCFDE, direct up-downconversion is assumed. QPSK modulation is used for both IO-SCFDE and CO-SCFDE.

Figure 18 shows the experimental setup for CO-SCFDE transmission using direct up-downconversion architecture. The experimental setup is similar to our previous work [13]. At the transmit side, an AWG at $10 \mathrm{GS} / \mathrm{s}$ generates the real and imaginary components of the QPSK signal to drive an optical I/Q modulator [13]. A recirculating loop consisting of one span of $100 \mathrm{~km}$ SSMF and an optical amplifier is used for longhaul transmission emulation. At the receive side, direct downconversion is employed by using an optical hybrid, two balanced receivers and a local oscillator laser. The polarization of the local laser is manually aligned to that of the incoming optical signal. The two outputs from the receivers representing I/Q components are sampled by a real-time sampling scope at $20 \mathrm{GS} / \mathrm{s}$. The sampling traces are uploaded into a computer for signal processing. Note the maximum bit rate is limited by the bandwidth and sampling rate of AWG.

At the transmit side, the QPSK waveform is first generated digitally and grouped by 128 QPSK symbols per block (or frame). The cyclic prefix, 1/16 of one block, is then inserted for each block. Additional sequences for frame synchronization and channel estimation are also inserted. There are many methods of performing channel estimation and the basic idea is to transmit a known sequence as preamble [49], and in this work we use the Chu sequence that has a flat magnitude in both the time and frequency domains $[47,49]$. The digital waveform is uploaded on AWG and converted into a time-domain analog waveform with two samples per symbol. The effective bit rate is $9.4 \mathrm{Gbits} / \mathrm{s}$, given that the sampling rate is $10 \mathrm{GS} / \mathrm{s}$. Note that the AWG may be replaced with conventional pattern generators since the digital waveform has only a few discrete levels.

At the receive side, the downconverted $\mathrm{RF}$ signal is first processed for the frame synchronization, namely, DFT window synchronization as in OFDM systems. The sequence is converted to the frequency domain through DFT, where the channel esti-

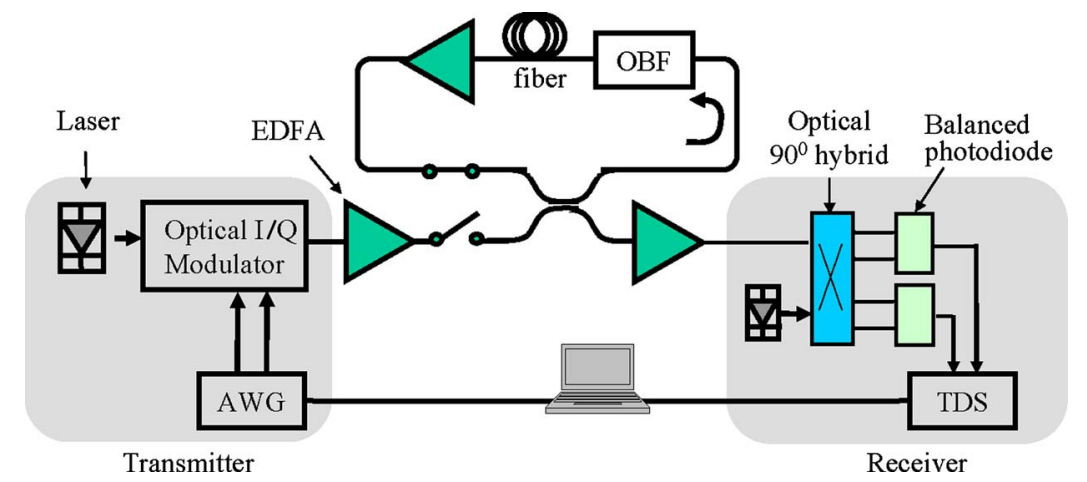

Fig. 18. Experimental setup for CO-SCFDE transmission using direct updownconversion architecture. 


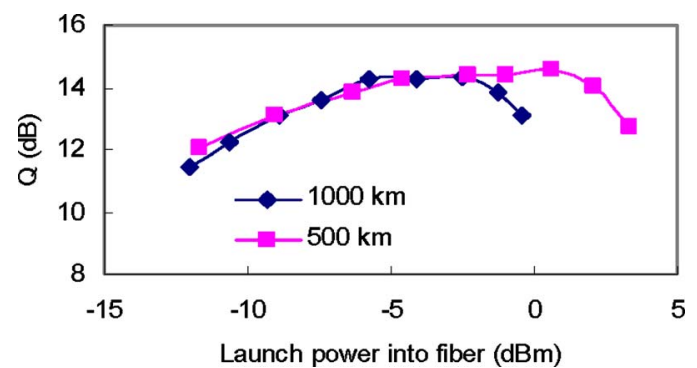

Fig. 19. Performance of CO-SCFDE as a function of launch power.

mation is conducted by comparing the transmitted and received Chu sequence [49]. After channel compensation and channel equalization, the signal is converted back to the time domain through IDFT. We then resample the sequence to one sample per symbol and carry out the phase estimation in the time domain, which is based on the $M$ th power-law method. The signal stream is grouped as blocks a manner similar to OFDM symbols.

We first measure the nonlinearity tolerance of single-carrier transmission by varying the launch power into each span. Figure 19 shows the measured Q factors at 500 and $1000 \mathrm{~km}$. For the $500 \mathrm{~km}$ transmission, the maximum Q factor is achieved at $\sim 1 \mathrm{dBm}$ launch power. For the $1000 \mathrm{~km}$ transmission, the optimum launch power is approximately $-3 \mathrm{dBm}$.

Figure 20(a) shows the RF spectrum measured after $1000 \mathrm{~km}$ transmission. The spectrum is simply that of a standard $10 \mathrm{Gbit} / \mathrm{s}$ QPSK signal. Figure 20(b) shows the BER measurement results after $1000 \mathrm{~km}$ transmission. At lower launch power, the system still has less than $2 \mathrm{~dB}$ of OSNR penalty, which may come from the imperfect channel estimation and compensation. When the launch power is $-0.1 \mathrm{dBm}$, the OSNR penalty is $\sim 3 \mathrm{~dB}$.

It can be seen that CO-SCFDE can achieve almost the same transmission performance of CO-OFDM. Let us briefly compare the CO-SCFDE and CO-OFDM in the following aspects:

(a) Computation complexity. The computation complexity of CO-SCFDE involving DFT and IDFT is the same as CO-OFDM, which scales as

$$
C_{\text {bit }} \propto \log _{2}\left(N_{s c}\right), \quad N_{s c}=\alpha D B,
$$

where $C_{\text {bit }}$ is the computation complexity defined as the number of multiplications required per bit, $N_{s c}$ is the number of subcarriers in CO-OFDM or the number of points used in DFT-IDFT in each CO-SCFDE block, $D$ is the accumulated channel $\mathrm{CD}, B$ is the baud rate, $\alpha$ is the proportional constant. It is of importance to point out that for SCTDE systems [10-12,22] based on FIR equalization, the computation complexity scales as $[10,12]$

$$
C_{\text {bit }} \propto D B^{2} .
$$

It can be seen that both OFDM and SCFDE are much superior to SCTDE in their scalability with both dispersion and bit rate. For this particular reason, we believe that CO-OFDM and CO-SCFDE are attractive choices of modulation format for nextgeneration $100 \mathrm{Gbit} / \mathrm{s}$ Ethernet transmission.

(a)

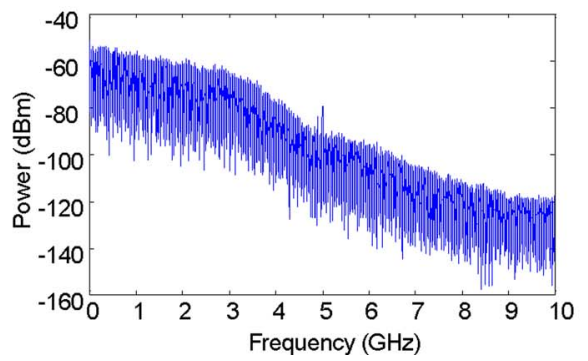

(b)

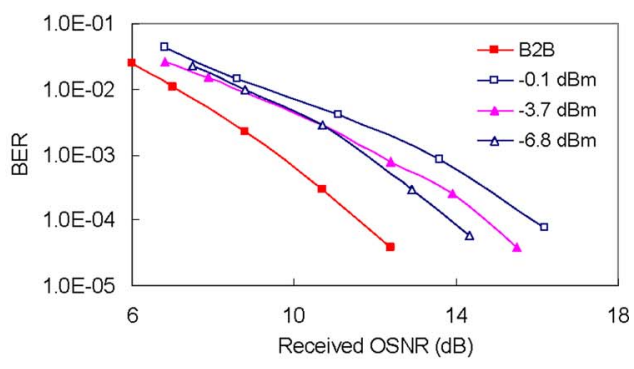

Fig. 20. (a) RF spectrum of the received CO-SCFDE signal and (b) BER sensitivity with varying launch powers. 
(b) System nonlinearity. Although the CO-SCFDE is superior in the electrical OFDM transmitter design due to smaller PAPR and less fiber nonlinearity at the start of the fiber link, the difference will diminish considering that the fiber link may not be chromatic dispersion compensated, and the PAPR will regrow in CO-SCFDE systems. By using proper clipping, or pre- or post-linearity compensation [13,50], we anticipate that the difference between CO-SCFDE and CO-OFDM is insignificant.

(c) Bit and power loading. One of the major advantages for CO-OFDM is the ability of frequency-domain management at the transmitter, involving bit and power loading. How this capability is to be exploited in the optical domain is of great research interest. Because coherent direction is used in both schemes, the channel response is a superposition of the electrical channel and the optical channel. Therefore, any severe fading from either the RF or the optical channel can be effectively mitigated through bit and power loading in CO-OFDM systems.

(d) Spectral efficiency. The CO-OFDM spectrum is inherently tighter than SCFDE. This suggests that both electrical and optical filters are easier to produce and the penalty as a result of tight spectral filtering is much less in CO-OFDM than in CO-SCFDE.

(e) Sampling rate. The SCFDE sampling rate is preferred to be double the baud rate, as it is sensitive to the sampling phase for a lower sampling rate. Although resampling can resolve this problem, it requires precise timing alignment, and also resampling is computation intensive. In CO-OFDM, oversampling is simply done by not filling the edge subcarriers in order to tight bound the signal spectrum [13], and therefore only $10 \%-20 \%$ oversampling is sufficient.

(f) Subcarrier or bandwidth allocation for performance monitoring and multiaccess. It is a great advantage to place DFT in the transmitter for CO-OFDM systems. The ability and flexibility to allocate a certain number subcarriers for channel estimation and performance monitoring will prove to be an attractive feature for CO-OFDM. For instance, this leaves the option of grouping a band of subcarriers for monitoring, which can be easily detected without processing the entire spectrum. Similarly, the grouping of subcarriers allows for the dynamic bandwidth allocation for multiaccess networks using the orthogonal frequency-domain-multiple-access (OFDMA) scheme [20]. All these are difficult to achieve for the CO-SCFDE system.

\section{Matching the Design to the Technology}

All the studies so far on CO-OFDM have been proof of concept in nature, either in the form of theoretical analysis or the experimental demonstration using off-line processing $[3,4,13,14]$. Nevertheless, a technological analysis in conjunction with those studies is able to reveal what the current and emerging technologies will entail for CO-OFDM implementation. In this section, we will list the components or subsystems required for the design of $40 \mathrm{Gbit} / \mathrm{s}$ CO-OFDM, based on recent theoretical analysis and experimental demonstration, and show how they match with today's technology.

(a) Optoelectronic components. From the $2 \times 2$ MIMO-OFDM experiment in Subsection 4.D, the transmit and receive bandwidth required for $40 \mathrm{Gbit} / \mathrm{s}$ CO-OFDM is $\sim 6 \mathrm{GHz}$. These components are commercially available including an optical I/Q modulator, balanced photodiodes, and an associated linear RF amplifier driver and post RF amplifier.

(b) Optical components. The optical components required for $2 \times 2$ MIMO-OFDM include lasers, a $90^{\circ}$ optical hybrid, and a polarization beam combiner-splitter. The linewidth for the transmit and receive laser is preferably in the range of $100 \mathrm{kHz}$, which is already in mass production by Intel. More advanced algorithms may relax the linewidth requirement to $1 \mathrm{MHz}$ and above [4], similar to single-carrier systems. The $90^{\circ}$ optical hybrid can be constructed using fused fiber technology, implying a potentially cost-effective solution [10]. There had been significant effort for integrated coherent circuits more than a decade ago, for instance, [51] reported an optical coherent receiver module that includes an optical $90^{\circ}$ hybrid fabricated by the planar lightwave circuit (LC) technique, two twin p-i-n photodetectors, and two DC-coupled preamplifiers. We believe this photonic integration circuit (PIC) will greatly accelerate the acceptance of CO-OFDM systems through integrating a PBS, an optical hybrid, a balanced receiver, and LO lasers in one or multiple PIC chips.

(c) Signal processing elements. CO-OFDM requires a digital signal processing (DSP) chip with an IFFT-FFT engine and DAC-ADC elements. Although no commer- 
cial DAC-ADC chips provide $6 \mathrm{GHz}$ bandwidth, the recent progress made in the highspeed CMOS ASIC chips is very encouraging [22]. In that report, the signal processing chip including both DSP and DAC-ADC contains $20 \times 10^{6}$ gates, four six-bit ADCs at $20 \mathrm{Gbits} / \mathrm{s}$, operating at 12 tera operations per second on a $90 \mathrm{~nm}$ platform [22]. Since the computation complexity of CO-OFDM is lower than the FIR-filter-based singlecarrier scheme [52], it is reasonable to conclude that the current silicon technology suffices to support $40 \mathrm{Gbit} / \mathrm{s}$ CO-OFDM systems. It is noted that signal processing in CO-OFDM is riding on Moore's law. Given the fact that Intel will soon produce their next-generation Penryn CPUs from their forthcoming $45 \mathrm{~nm}$ manufacturing process based on high- $k$ technology [53], the CMOS ASIC chip for CO-OFDM can potentially migrate to the same process in the near future. In so doing, the energy consumption of $45 \mathrm{~nm}$ processing is approximately $1 / 8$ of $90 \mathrm{~nm}$ processing, assuming the same number of operations per second needed for the chip, or the capability of the chip, namely, the number of operations per second for the chip, will increase eightfold due to the increase of the switching speed as well as a greater number of gates that can be placed on chip, assuming the same size for the chip. The silicon technology rapidly advancing well into the next decade will no doubt fuel this unmistakable trend in optical communications so that more and more functionalities will be shifted to these high-speed CMOS chips.

\section{Conclusion}

In this paper, we have reviewed the progress on CO-OFDM systems as well as presented the latest research results. We first laid out the signal processing aspect of the CO-OFDM and then showed a $2 \times 2$ multiple-input-multiple-output OFDM (MIMOOFDM) representation for the single-mode fiber optical channel. We further showed and discussed experimental demonstrations for various MIMO-OFDM configurations. In particular, we carried out long-haul transmission for the $2 \times 2$ and $1 \times 2$ MIMOOFDM systems and short-reach transmission for the $2 \times 1$ MIMO-OFDM systems that may have promise for optical broadcasting networks. Finally, another class of the frequency-domain equalization (FDE) technique, namely, incoherent or coherent optical single-carrier frequency-domain equalization (IO-SCFDE or CO-SCFDE) was discussed and demonstrated.

\section{Acknowledgment}

This work was supported by the Australian Research Council (ARC).

\section{References}

1. W. Shieh and C. Athaudage, "Coherent optical orthogonal frequency division multiplexing," Electron. Lett. 42, 587-589 (2006).

2. W. Shieh, W. Chen, and R. S. Tucker, "Polarization mode dispersion mitigation in coherent optical orthogonal frequency division multiplexed systems," Electron. Lett. 42, 996-997 (2006).

3. W. Shieh, X. Yi, and Y. Tang, "Transmission experiment of multi-gigabit coherent optical OFDM systems over 1000 km SSMF fiber," Electron. Lett. 43, 183-185 (2007).

4. S. L. Jansen, I. Morita, N. Takeda, and H. Tanaka, "20-Gb/s OFDM transmission over 4,160-km SSMF enabled by RF-pilot tone phase noise compensation," in Optical Fiber Communication Conference and Exposition and the National Fiber Optic Engineers Conference, Technical Digest (Optical Society of America, 2007), paper PDP15.

5. L. Kazovsky, "Multichannel coherent optical communications systems," J. Lightwave Technol. 5, 1095-1102 (1987).

6. T. Okoshi, "Heterodyne and coherent optical fiber communications: recent progress," IEEE Trans. Microwave Theory Tech. 82, 1138-1149 (1982).

7. J. M. Kahn, I. M. I. Habbab, and C. R. Giles, "1 Gbit/s zero-IF DPSK coherent optical system using a single photodetector," Electron. Lett. 24, 1455-1457 (1988).

8. A. H. Gnauck et al., " $2.5 \mathrm{~Tb} / \mathrm{s}(64 \times 42.7 \mathrm{~Gb} / \mathrm{s})$ transmission over $40 \times 100 \mathrm{~km}$ NZDSF using RZ-DPSK format and all-Raman-amplified spans," in Optical Fiber Communication Conference and Exposition, Technical Digest (Optical Society of America, 2002), paper FC2.

9. D. S. Ly-Gagnon, S. Tsukarnoto, K. Katoh, and K. Kikuchi, "Coherent detection of optical quadrature please-shift keying signals with carrier phase estimation," J. Lightwave Technol. 24, 12-21 (2006).

10. S. J. Savory, G. Gavioli, R. I. Killey, and P. Bayvel, "Electronic compensation of chromatic dispersion using a digital coherent receiver," Opt. Express 15, 2120-2126 (2007).

11. C. Laperle, B. Villeneuve, Z. Zhang, D. McGhan, H. Sun, and M. O'Sullivan, "Wavelength 
division multiplexing (WDM) and polarization mode dispersion (PMD) performance of a coherent $40 \mathrm{Gbit} / \mathrm{s}$ dual-polarization quadrature phase shift keying (DP-QPSK) transceiver," in Optical Fiber Communication Conference and Exposition and the National Fiber Optic Engineers Conference, Technical Digest (Optical Society of America, 2007), paper PDP16.

12. G. Charlet, J. Renaudier, M. Salsi, H. Mardoyan, P. Tran, and S. Bigo, "Efficient mitigation of fiber impairments in an ultra-long haul transmission of $40 \mathrm{Gbit} / \mathrm{s}$ polarizationmultiplexed data, by digital processing in a coherent receiver," in Optical Fiber Communication Conference and Exposition and the National Fiber Optic Engineers Conference, Technical Digest (Optical Society of America, 2007), paper PDP17.

13. W. Shieh, X. Yi, Y. Ma, and Y. Tang, "Theoretical and experimental study on PMD-supported transmission using polarization diversity in coherent optical OFDM systems," Opt. Express 15, 9936-9947 (2007).

14. S. L. Jansen, I. Morita, and H. Tanaka, " $16 \times 52.5-\mathrm{Gb} / \mathrm{s}, 50-\mathrm{GHz}$ spaced, POLMUX-COOFDM transmission over $4,160 \mathrm{~km}$ of SSMF enabled by MIMO processing KDDI R\&D Laboratories," presented at the European Conference on Optical Communications, Berlin, Germany, September 16-20, 2007, paper PD1.3.

15. J. M. Tang, P. M. Lane, and K. A. Shore, " $30 \mathrm{~Gb} / \mathrm{s}$ transmission over $40 \mathrm{~km}$ directly modulated DFB laser-based SMF links without optical amplification and dispersion compensation for VSR and metro applications," in Optical Fiber Communication Conference (Optical Society of America, 2006), paper JThB8.

16. I. B. Djordjevic and B. Vasic, "Orthogonal frequency division multiplexing for high-speed optical transmission,” Opt. Express 14, 3767-3775 (2006).

17. O. Gonzalez, R. Perez-Jimenez, S. Rodriguez, J. Rabadan, and A. Ayala, "OFDM over indoor wireless optical channel,” IEE Proc.: Optoelectron. 152, 199-204 (2005).

18. A. J. Lowery, L. Du, and J. Armstrong, "Orthogonal frequency division multiplexing for adaptive dispersion compensation in long haul WDM systems," presented at the Optical Fiber Communication Conference, Anaheim, Calif., March 5-10, 2006, paper PDP39.

19. S. C. J. Lee, F. Breyer, S. Randel, M. Schuster, J. Zeng, F. Huijskens, H. P. A. van den Boom, A. M. J. Koonen, and N. Hanik, "24-Gbls transmission over $730 \mathrm{~m}$ of multimode fiber by direct modulation of an 850-nm VCSEL using discrete multi-tone modulation," in Optical Fiber Communication Conference (IEEE, 2007), paper PDP6.

20. D. Qian, J. Hu, J. Yu, P. Ji, L. Xu, T. Wang, M. Cvijetic, and T. Kusano, "Experimental demonstration of a novel OFDM-A based $10 \mathrm{~Gb} / \mathrm{s}$ PON architecture," presented at the European Conference on Optical Communications, Berlin, Germany, September 16-20, 2007, paper 5.4.1.

21. S. Hara and R. Prasad, Multicarrier Techniques for 4G Mobile Communications (Artech House, 2003).

22. K. Roberts, "Electronic dispersion compensation beyond $10 \mathrm{~Gb} / \mathrm{s}$ " in Technical Digest, LEOS Summer Topical Meeting (IEEE, 2007), paper MA2.3.

23. J. Sitch, "Implementation aspects of high-speed DSP for transmitter and receiver signal processing," in Technical Digest, LEOS Summer Topical Meeting (IEEE, 2007), paper MA4.3.

24. W. Shieh, H. Bao, and Y. Tang, "Coherent optical OFDM: theory and design," Opt. Express 16, 841-859 (2008).

25. Y. Tang, W. Shieh, X. Yi, and R. Evans, "Optimum design for RF-to-optical up-converter in coherent optical OFDM systems," IEEE Photon. Technol. Lett. 19, 483-485 (2007).

26. H. Bao and W. Shieh, "Transmission simulation of coherent optical OFDM signals in WDM systems," Opt. Express 15, 4410-4418 (2007).

27. I. B. Djordjevic, "PMD compensation in fiber-optic communication systems with direct detection using LDPC-coded OFDM," Opt. Express 15, 3692-3701 (2007).

28. L. Hanzo, M. Munster, B. J. Choi, and T. Keller, OFDM and MC-CDMA for Broadband Multi-User Communications, WLANs and Broadcasting (Wiley, 2004).

29. T. M. Schmidl and D. C. Cox, "Robust frequency and timing synchronization for OFDM," IEEE Trans. Commun. 45, 1613-1621 (1997).

30. E. Ip, J. P. Kahn, D. Anthon, and J. Hutchins, "Linewidth measurements of MEMS-based tunable lasers for phase-locking applications," IEEE Photon. Technol. Lett. 17, 2029-2031 (2005).

31. H. Sari, G. Karam, and I. Jeanclaude, "Transmission techniques for digital terrestrial TV broadcasting," IEEE Commun. Mag. 33(2), 100-109 (1995).

32. P. Moose, "A technique for orthogonal frequency division multiplexing frequency offset correction," IEEE Trans. Commun. 42, 2908-2914 (1994).

33. S. Hara, M. Mouri, M. Okada, and N. Morinaga, "Transmission performance analysis of multi-carrier modulation in frequency selective fast Rayleigh fading channel," Wireless Personal Commun. 2, 335-356 (1996).

34. X. Yi, W. Shieh, and Y. Tang, "Phase estimation for coherent optical OFDM," IEEE Photon. Technol. Lett. 19, 919-921 (2007).

35. S. Wu and Y. Bar-Ness, "A phase noise suppression algorithm for OFDM-based WLANs," IEEE Commun. Lett. 6, 535-537 (2002).

36. N. Gisin and B. Huttner, "Combined effects of polarization mode dispersion and polarization dependent losses in optical fibers," Opt. Commun. 142, 119-125 (1997).

37. H. Bolcskei, D. Gesbert, and A. J. Paulraj, "On the capacity of OFDM-based spatial multiplexing systems," IEEE Trans. Commun. 50, 225-34 (2002).

38. Y. Han and G. Li, "Coherent optical communication using polarization multiple-inputmultiple-output,” Opt. Express 13, 7527-7535 (2005). 
39. W. Shieh, "PMD-supported coherent optical OFDM systems," IEEE Photon. Technol. Lett. 19, 134-136 (2007)

40. W. Shieh, "Coherent optical MIMI-OFDM for optical fibre communication systems," presented at Workshop 5, European Conference on Optical Communications, Berlin, Germany, September 16-20, 2007.

41. N. Cvijetic, L. Xu, and T. Wang, "Adaptive PMD compensation using OFDM in long-haul $10 \mathrm{~Gb} / \mathrm{s}$ DWDM systems," in Optical Fiber Communication Conference and Exposition and the National Fiber Optic Engineers Conference, Technical Digest (Optical Society of America, 2007), paper OTuA5.

42. M. Mayrock and H. Haunstein, "PMD tolerant direct-detection optical OFDM system," presented at the European Conference on Optical Communication (ECOC), September 16-20, Berlin, Germany, paper 5.2.5.

43. L. Moller, Y. Su, and G. Raybon, "Polarization-mode-dispersion-supported transmission in 40-Gb/s long haul systems," IEEE Photon. Technol. Lett. 15, 335-337 (2003).

44. S. M. Alamouti, "A simple transmit diversity technique for wireless communications," IEEE J. Sel. Areas Commun. 16, 1451-1458 (1998).

45. Y. Han and G. Li, "Polarization diversity transmitter and optical nonlinearity mitigation using polarization-time coding," presented at OSA Topical Meeting, Coherent Optical Technologies and Applications, Whistler, Canada, June 25-30, 2006, paper CThC7.

46. S. M. Lee, S. G. Mun, M. H. Kim, and C. H. Lee, "Demonstration of a long-reach DWDM-PON for consolidation of metro and access networks," J. Lightwave Technol. 25, 271-276 (2007).

47. D. Falconer, S. L. Ariyavisitakul, A. Benyamin-Seeyar, and B. Eidson, "Frequency domain equalization for single-carrier broadband wireless systems," IEEE Commun. Mag. 40(4), 58-66 (2002).

48. Z. Wang, X. Ma, and G. B. Giannakis, "OFDM or single-carrier block transmissions?" IEEE Trans. Commun. 52, 380-394 (2004).

49. D. C. Chu, "Polyphase codes with good periodic correlation properties," IEEE Trans. Inf. Theory 18, 531-532 (1972).

50. A. J. Lowery, "Fiber nonlinearity pre- and post-compensation for long-haul optical links using OFDM," Opt. Express 15, 12965-12970 (2007).

51. S. Norimatsu, N. Takachio, Y. Inoue, M. Hosoya, H. Tsunetsugu, and S. Hata, "An optical 90-degrees-hybrid balanced receiver module using a planar lightwave circuit," IEEE Photon. Technol. Lett. 6, 737-740 (1994).

52. H. Bulow, "Electronic dispersion compensation," in Optical Fiber Communication Conference and Exposition and the National Fiber Optic Engineers Conference, Technical Digest (Optical Society of America, 2007), paper OMG5.

53. M. T. Bohr, R. S. Chau, T. Ghani, and K. Mistry, "The high- $k$ solution," IEEE Spectrum 44(10), 29-35 (2007). 